International Journal of Pure and Applied Mathematics

Volume 84 No. 1 2013, 29-63

ISSN: 1311-8080 (printed version); ISSN: 1314-3395 (on-line version)

url: http://www.ijpam.eu

doi: http://dx.doi.org/10.12732/ijpam.v84i1.3

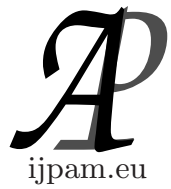

\title{
AN EXPLORATION ON GOLDBACH'S CONJECTURE
}

\author{
E. Markakis ${ }^{1}$, C. Provatidis ${ }^{2 \S}$, N. Markakis ${ }^{3}$ \\ ${ }^{1}$ Vassilissis Olgas 129B \\ 54643, Thessaloniki, GREECE \\ ${ }^{2}$ National Technical University of Athens \\ 9, Heroes of Polytechnion Ave., Zografou Campus \\ 157 80, Athens, GREECE \\ ${ }^{3}$ Cram School "Methodiko" \\ Vouliagmenis and Kyprou 2, \\ 16452 Argyroupolis, GREECE
}

\begin{abstract}
This paper divides the set of natural numbers in six equivalence classes and determines two of them as candidate to include all prime numbers. Concerning the even numbers themselves, these were divided into three subsets using a basic cell $(6 n-2,6 n$ and $6 n+2)$. Based on the aforementioned tools, this paper proposes a deterministic process of finding all pairs $(p, q)$ of odd numbers (composites and primes) of natural numbers $\geq 3$ whose sum $(p+q)$ is equal to a given even natural number $2 n \geq 6$. Based on this procedure and also relying on the distribution of primes in the set $\mathbb{N}$ of natural numbers, a closed analytical formula is proposed for the estimation of the number of primes that satisfy Goldbach's conjecture for positive integers $\geq 6$.
\end{abstract}

AMS Subject Classification: 11-XX

Key Words: Goldbach's conjecture, prime numbers, statistics

Received: September 27, 2012

(c) 2013 Academic Publications, Ltd. url: www.acadpubl.eu

${ }^{\S}$ Correspondence author 


\section{Introduction}

As is known, on June 7, 1742, Christian Goldbach in a letter to Leonhard Euler (see [1]) argued that, every even natural number greater than 4 can be written as a sum of two primes, namely:

$$
2 n=p+q \text {, where } n>2, \text { and } p, q \text { are prime numbers. }
$$

To save space, we do not use the original wording of that old time, but we focus our attention on the modified formula (1) which is consistent with the newest definition of the set of prime numbers $\mathbb{P}=\{2,3,5,7,11, \ldots\}$, which does not include the unit.

On August 8, 1900, David Hilbert gave a famous speech during the second International Congress of Mathematics in Paris, in which he proposed 23 problems for mathematicians of the 20th century, including Goldbach's conjecture (see [2]). Later, in 1912, Landau sorted four main problems for the prime numbers including Goldbach's conjecture (see [3],[4]). The first scientific work on Goldbach's conjecture was made in the 1920's. Note that in 1921, Hardy said that Goldbach's conjecture is not only the most famous and difficult problem in number theory, but the whole of mathematics.

It is known that the most difficult so-called strong Goldbach conjecture was preceded by important work in the so-called weak Goldbach conjecture. The weak conjecture, which is known as the odd Goldbach conjecture or ternary Goldbach problem or 3-primes problem, stated that: any number greater than 7 can be expressed as a sum of three primes (one prime number can be used more than once in the same sum). The above assumption is called "weak" because if the strong Goldbach's conjecture (which concerns sums of two primes) is proved, then the weak will be true.

The weak formulation of the conjecture has not been yet proven, but there have been some useful although somewhat failed attempts. The first of these works was in 1923 when, using the "circle method" and assuming the validity of the hypothesis of a generalized Riemann, Hardy and Littlewood (see [5]) proved that every sufficiently large odd integer is sum of three odd primes and almost all the even number is the sum of two primes. In 1919, Brun (see [6]), using the method of his sieve proved that every large even number is the sum of two numbers each of whom has at least nine factors of primes. Then in 1930, using the Brun's method along with his own idea of "density" of a sequence of integers, Schnirelman (see [7]) proved that every sufficiently large integer is the sum of maximum $c$ primes for a given number $c$. Then in 1937, Vinogradov (see [8]), using the circle method and his own method to estimate the expo- 
nential sum in a variable prime number, was able to overcome the dependence of the great Riemann hypothesis and thus provide the evidence of the findings of Hardy and Littlewood now without conditions. In other words, he directly proved (Vinogradov's theorem) that all "sufficiently large" odd number can be expressed as the sum of three primes. The original proof of Vinogradov, based on inefficient theorem of Siegel-Walfisz, did not put a limit for the term "sufficiently large" while his student Borozdkin (see [9]) showed in 1956 that $n_{0}=3^{3^{15}}=3^{14348907}$ is sufficiently large (has 6,846,169 digits). Later, after improvements in the method of Brun, in 1966 Jing-run Chen (see [10]) managed to prove that every large integer is the sum of a prime and a product of at most two primes. In 2002, Liu and Wang (see [11]) lowered the threshold around $n>e^{3100} \approx 2 \times 10^{1346}$. The exponent is too large to allow control of all smaller numbers with the assistance of a digital computer. According to Internet reports $[12,13]$, the computer assisted search arrived for the strong Goldbach conjecture up to order $10^{18}$ (http://www.ieeta.pt/ tos/goldbach.html) and, for the weak Goldbach conjecture not much more. In 1997, Deshouillers et al. (see [14]) showed that the generalized Riemann hypothesis implies the weak Goldbach's conjecture for all numbers. Also, Kaniecki (see [15]) showed that every odd number is the sum of at most five primes, provided the validity of Riemann Hypothesis.

Most of these classic works have been included in a collective volume by Wang (see [16]). Specifically, in that volume Section 1 includes the representation of an odd number as a sum of three primes in six papers (Hardy and Littlewood; Vinogradov; Linnik; Pan; Vaughan; Deshouillers et al.), Section 2 includes the representation of an even number as a sum of two nearly primes in six other works of (Brun; Buchstab; Kuhn; Selberg; Wang; Selberg) and finally Section 3 includes the representation of an even number as a sum of a prime and an almost prime in nine works (Renyi; Wang; Pan; Barban Til; Buchstab; Vinogradov; Bombieri; Chen; Pan). Finally, apart from the individual reports of certain articles, the collective volume includes 234 additional citations arranged by author, referring to the period 1901-2001.

The strong formulation of Goldbach conjecture, which is the subject of this paper, is much more difficult than the above weak one. Using the above method of Vinogradov (see [8]), in separate works Chudakov (see [17]), van der Corput (see [18]) and Estermann (see [19]) showed that almost all even number can be written as a sum of two primes (in the sense that the fraction of even number tends to the unit). As mentioned above, in 1930, Schnirelman (see [7]) showed that every even number $n \geq 4$ can be written as a sum of at most 20 primes. This result in turn enriched by other authors; the most well-known result due to 
Ramaré (see [20]) who in 1995 showed that every even number $n \geq 4$ is indeed a maximum sum of 6 primes. Indeed, resolving the weak Goldbach conjecture will come through that every even number $n \geq 4$ is the sum of at most 4 primes (see [21]). In 1973, using sieve theory methods Jing-run Chen (see [22]) showed that every sufficiently large even number can be written as a sum either of two primes or of one prime and one semiprime (i.e. a product of two primes), e.g. $100=23+7 \cdot 11$. In 1975, Montgomery and Vaughan (see [23]) showed that "most" even number is a sum of two primes. In fact, they showed that there were positive constants $c$ and $C$ such that for all sufficiently large numbers $N$, every even number less than $N$ is the sum of two primes with $C N^{1-c}$ exceptions at the most. In particular, all the even integers that are not sum of two primes have zero density. Linnik (see [24]) proved, in 1951, the existence of a constant $K$ such that every sufficiently large even number is the sum of two primes and a maximum of $K$ powers of 2. Heath-Brown and Puchta (see [25]) in 2002 found that the value $K=13$ works well. The latter improved to $K=8$ by Pintz and Ruzsa (see [26]) in 2003.

It is noteworthy that, in 2000, the relation (1) was verified using computers for even numbers up to $4 \times 10^{16}$ (see [27]), and the attempt was repeated by T. Oliveira e Silva with the help of distributed computing network to $n \leq 1.609 \times$ $10^{18}$ and in selected areas up to $4 \times 10^{18}$ (see [13]). However, mathematically these checks do not constitute conclusive evidence of validity of (1), and the effort continues today [28].

In addition to the above papers, the interested reader can consult internet sources [29-32]. Finally, Goldbach's conjecture has been the subject of statistical approach [33], education [34], as well as narrative storytelling and popular books [35-37].

In this paper we present a theoretical framework that provides an estimate of the number of prime numbers satisfying $\mathrm{Eq}(1)$.

\section{A Deterministic Procedure for the Representation of an Even Number as Sum of Two Odds}

By the definition of prime numbers, $\mathbb{P}=\{2,3,5,7,11, \ldots\}$, since they are divisible only by themselves and the unit, it follows that "the only even prime number is 2 ". If for a moment we assume that $p=2$, then the only case that fulfils $\operatorname{Eq}(1)$ is when $q=2$, otherwise the resulting sum would be equal to an odd number. Because the resulting even number 4 (i.e. $n=2$ ) is outside the scope of interest (we care only for $n>2$ ), it is obvious that $\operatorname{Eq}(1)$ makes sense only 
for the odd primes, i.e. for all primes greater than 2, which obviously comprise the set $\mathbb{P}-\{2\}$.

In Subsections 2.1 and 2.2 we quote useful tools (for later use) and then in 2.3 we present a series of useful theorems.

\subsection{Equivalence Classes}

As known, an equivalence relation on the set $\mathrm{A}$, such as e.g. $K(\alpha)=\{x / x \in$ $A \wedge x R \alpha\}$ divides the set A into subsets, named equivalence classes, which are disjoint to each other and their union gives $\mathrm{A}$.

Suppose $\mathbb{Z}$ is the set of integer numbers, $\mathbb{Z}^{2}$ its Cartesian (tensor) product and $\mathrm{R}$ is the binary relation on the set $\mathbb{Z}^{2}$, which is defined as follows:

$$
R=\left\{(\alpha, \beta) /(\alpha, \beta) \in \mathbb{Z}^{2} \text { and }(\alpha-\beta) \text { is divisible by } n \in \mathbb{N}\right\}
$$

that is $(\alpha-\beta)=\lambda n$ or $\alpha=\lambda n+\beta$ (the identity of division) and $\mathbb{N}$ is the set of natural numbers.

The binary relation $\mathrm{R}$ is an equivalence relation because, as can be proven, is reflexive, symmetric and transitive. The natural number $n$ determines the number of equivalence classes.

If we take the set of natural numbers $\mathbb{N}$ and define the divisor $n=6$, then $\mathbb{N}$ is divided into six equivalence classes, those of elements $0,1,2,3,4$ and 5 , namely:

$$
\begin{aligned}
& K(0)=\{x / x=6 \lambda+0, \lambda \in \mathbb{N}\} \\
& K(1)=\{x / x=6 \lambda+1, \lambda \in \mathbb{N}\} \\
& K(2)=\{x / x=6 \lambda+2, \lambda \in \mathbb{N}\} \\
& K(3)=\{x / x=6 \lambda+3, \lambda \in \mathbb{N}\} \\
& K(4)=\{x / x=6 \lambda+4, \lambda \in \mathbb{N}\} \\
& K(5)=\{x / x=6 \lambda+5, \lambda \in \mathbb{N}\}
\end{aligned}
$$

The above classes are disjoint each other and their union gives the set $\mathbb{N}$, that is:

$$
K(0) \cup K(1) \cup K(2) \cup K(3) \cup K(4) \cup K(5)=\mathbb{N}
$$

Obviously, the set of natural numbers corresponding to the equivalence classes $K(2)$ and $K(4)$ are divisible by $2 ; K(3)$ is divisible by 3 , and $K(0)$ is divisible by both 2 and 3 . Therefore the set of natural numbers contained in the equivalence classes $K(0), K(2), K(3)$ and $K(4)$ are composite numbers as being multiples of 2 and 3 . 
Conclusion-1: The remaining equivalence classes, $K(1)$ and $K(5)$, contain all primes (except of 2 and 3 ) as well as the multiples of primes being $>3$; these can be combined in the formula:

$$
\mathbf{6} \lambda \pm \mathbf{1}=\text { Primes }+ \text { Multiples of Primes }>\mathbf{3}
$$

In the representation of odd numbers according to $\mathrm{Eq}(5 \mathrm{a})$, we achieved to condense the feasible space of prime numbers in $\mathbb{N}$ by a factor of 3 (i.e. it is a subset of two classes out of six).

For the sake of uniformity, the multiples of 3 will be denoted, as appropriate, either as $6 \lambda-3$ for $\lambda=1,2,3, \ldots N$, or like $6 \lambda+3$ for $\lambda=0,1,2, \ldots N$, that is:

$$
6 \lambda \pm 3=\text { Multiples of } 3
$$

\subsection{Even Numbers}

From Number Theory we know that in any three consecutive even numbers one of them is a multiple of 3 , so this even number can be written as $3 \cdot 2 n=$ $6 n$. The triple of numbers $(6 n-2,6 n, 6 n+2)$ are consecutive even numbers, since they differ by 2 units. This implies that the subsets of even numbers $\{x / x \quad 6 n-2,6 n, 6 n+2, n \in \mathbb{N}\}$ are disjoint and their union is the set $\mathbb{N}_{2}$ of all even numbers $\geq 4$.

Conclusion-2: The set of even numbers $\geq 4$ can be denoted with:

$$
\mathbb{N}_{2}=\{x / x \quad 6 n-2,6 n, 6 n+2\} \text { where } n \geq 1,
$$

and the above triple of even numbers is the basic cell to create the even numbers, for $n \geq 1$, in the sequence of our work.

If we replace $n$ in $\operatorname{Eq}(6)$ with two natural numbers, i.e. $\lambda_{i}$ and $\lambda_{j}$, where $\left(\lambda_{i}, \lambda_{j}\right) \in \mathbb{N}^{2}$, such as:

$$
n=\lambda_{i}+\lambda_{j}
$$

the triple of the successive even numbers in the basic cell is written as:

$$
\begin{array}{r}
6\left(\lambda_{i}+\lambda_{j}\right)-2 \\
6\left(\lambda_{i}+\lambda_{j}\right) \\
6\left(\lambda_{i}+\lambda_{j}\right)+2 .
\end{array}
$$

Equations (8) may be further transformed as follows:

$$
6\left(\lambda_{i}+\lambda_{j}\right)-2=\left(6 \lambda_{i}-1\right)+\left(6 \lambda_{j}-1\right)
$$




$$
\begin{aligned}
6\left(\lambda_{i}+\lambda_{j}\right) & =\left(6 \lambda_{i}-1\right)+\left(6 \lambda_{j}+1\right), \\
6\left(\lambda_{i}+\lambda_{j}\right) & =\left(6 \lambda_{i}+1\right)+\left(6 \lambda_{j}-1\right), \\
6\left(\lambda_{i}+\lambda_{j}\right)+2 & =\left(6 \lambda_{i}+1\right)+\left(6 \lambda_{j}+1\right) .
\end{aligned}
$$

Conclusion-3: Thus symbolizing the even numbers, we managed to transform all the even numbers into a sum of two odd numbers of the form $6 \lambda_{i} \pm 1$, which, as proved, either are primes or multiples of primes $\geq 5$.

Of course the even numbers can be created also as a sum of two odd numbers of which either one or both be multiples of 3 . In this case however the even numbers of the form $6 n-2$ are generated solely as a sum according to $\mathrm{Eq}(10 \mathrm{a})$, the even numbers of the form $6 n$ can be created only as a sum of two odd multiples of 3 according to $\mathrm{Eq}(10 \mathrm{~b})$, whereas the even numbers of the form $6 n+2$ are generated solely as a sum according to $\mathrm{Eq}(10 \mathrm{c})$ :

$$
\begin{array}{r}
6 n-2=\left(6 \lambda_{i}+1\right)+\left(6 \lambda_{j}-3\right)=6\left(\lambda_{i}+\lambda_{j}\right)-2 \\
6 n=\left(6 \lambda_{i}+3\right)+\left(6 \lambda_{j}-3\right)=6\left(\lambda_{i}+\lambda_{j}\right) \\
6 n+2=\left(6 \lambda_{i}-1\right)+\left(6 \lambda_{j}+3\right)=6\left(\lambda_{i}+\lambda_{j}\right)+2 .
\end{array}
$$

Equations (10) imply that, for each even number the pairs of odd numbers, which are created on the basis of these equations, can give either zero or at most only one pair prime-to-prime.

More specifically, given that the set of odd numbers being multiples of 3 , $(6 \lambda \pm 3)$, from which the even numbers of the form $6 n$ are formed, only the number 3 is prime, this implies that under (10b), the only pair which verifies Goldbach's conjecture, is the " $3+3=6$ ".

Based on $\mathrm{Eq}(10 \mathrm{a})$, the even numbers of the form $6 n-2$ verify Goldbach's conjecture only if $\left(6 \lambda_{j}-3\right)=3$ while $\left(6 \lambda_{i}+1\right)=$ prime.

Finally, the even numbers of the form $6 n+2$ verify Goldbach's conjecture, by virtue of $(10 \mathrm{c})$, only if $\left(6 \lambda_{j}-3\right)=3$ while $\left(6 \lambda_{i}-1\right)=$ prime.

\subsection{Three Basic Theorems}

In the following we describe a deterministic procedure in which every even natural number can be decomposed into all possible sums of odd integers, i.e. primes or composites.

Theorem 1. Every even natural number $2 n$ (independently of its specific form $6 n-2,6 n, 6 n+2)$ can be decomposed into a sum of two odd natural numbers (primes or composites) in so many different ways, $n_{s}$, as the integer part (floor) of the rational number $(n-1) / 2$, that is $\lfloor(n-1) / 2\rfloor$. The index 
"s" stands for the word "sample", thus referring to a sample of $n_{s}$ odd numbers from which will be later choose the prime numbers.

Proof. We distinguish two cases.

1) When $n$ is odd, we form the sets:

$A=\{3,5, \ldots, n\}$ and $B=\{2 n-3,2 n-5, \ldots, n\}$. Since the order of items is not important in the sets, in order to maintain the desired sequence (in the form of rows or columns) we form the vectors $\vec{a}=[3,5, \ldots, n]$ and $\vec{b}=$ $[2 n-3,2 n-5, \ldots, n]$. It is obvious that all elements of the vector $\vec{c}=\vec{a}+\vec{b}$ are strictly defined and are equal to $2 n$ as opposed to probabilistic pairs that can be derived from the sets A and B. Also, it is evident that any enhancement of the vector $\vec{a}$ will give terms contained in the vector $\vec{b}$, displayed from right to left, so it makes no sense. Finally, it is obvious that the cardinality of two sets is the same, i.e. $\operatorname{card} A=\operatorname{card} B=(n-1) / 2$.

2) When $n$ is even, we form the sets:

$A=\{3,5, \ldots, n-1\}$ and $B=\{2 n-3,2 n-5, \ldots, n+1\}$. As previously, we consider the new vectors $\vec{a}^{\prime}=[3,5, \ldots, n-1]$ and $\vec{b}^{\prime}=[2 n-3,2 n-5, \ldots, n+1]$. It is obvious that all elements of the vector $\vec{c}^{\prime}=\vec{a}^{\prime}+\vec{b}^{\prime}$ are again equal to $2 n$. As previously, any enhancement of the vector $\vec{a}^{\prime}$ will give terms included into the vector $\vec{b}$, displayed from right to left. Finally, it is obvious that the cardinality of two sets is the same, i.e. $\operatorname{card} A=\operatorname{card} B=n / 2-1$.

Summarizing the results of the two above cases, it is easily concluded that:

$$
\operatorname{card} A=\operatorname{card} B=\lfloor(n-1) / 2\rfloor
$$

Conclusion-4: The higher an even number $2 n$ is, the higher the number of pairs of odd numbers $n_{s}$.

Theorem 2. If the $n_{s}$ pairs of odd numbers $\left(p_{s}, q_{s}\right)$ involved in Theorem1 , whose sum is equal to the even number $2 n$, are plotted in orthocanonical system of axes $p_{s} q_{s}$, they will belong to a straight line which forms 45 degrees to both axes $p_{s}$ and $q_{s}$.

Proof. From Analytical Geometry we know that in a $x-y$ system, every straight line intersecting the $x$ axis at the point $A(\alpha, 0)$ and the $y$ axis at the point $B(0, \beta)$ satisfies the equation: $x / \alpha+y / \beta=1$. In our specific case, if we select the $x$ axis to represent the term $p_{s} \in \mathbb{N}_{0}$, while $y$ axis to represent the term $q_{s} \in \mathbb{N}_{0}$, then obviously it holds $\alpha=\beta=2 n$ (see Figure 1 ). It is also obvious that the $(2 n+1)$ integers of the interval $[0, \alpha]$ and the corresponding $(2 n+1)$ 
integers of the interval $[0, \beta]$, of which sum equals to $2 n$, correspond to $(2 n+1)$ discrete points along the straight segment AB. Given that we are interested in only the odd numbers $p_{s}, q_{s}>3$ which satisfy $\mathrm{Eq}(1)$, without necessarily being prime numbers, we must leave out the three pairs being closest to the $x$ axis: $(2 n, 0),(2 n-1,1),(2 n-2,2)$, as well as the three pairs closest to the $y$ axis: $(0,2 n),(1,2 n-1),(2,2 n-2)$. These six points are denoted into Figure 1 by the symbol $(\times)$. Therefore, the number of candidate points for further examination is $n_{s}=\lfloor(n-1) / 2\rfloor$, two of which coincide with the ends $\mathrm{C}$ and $\mathrm{D}$ (note that the set of all discrete points/pairs that correspond to even and odd integers is $2 n-5)$.

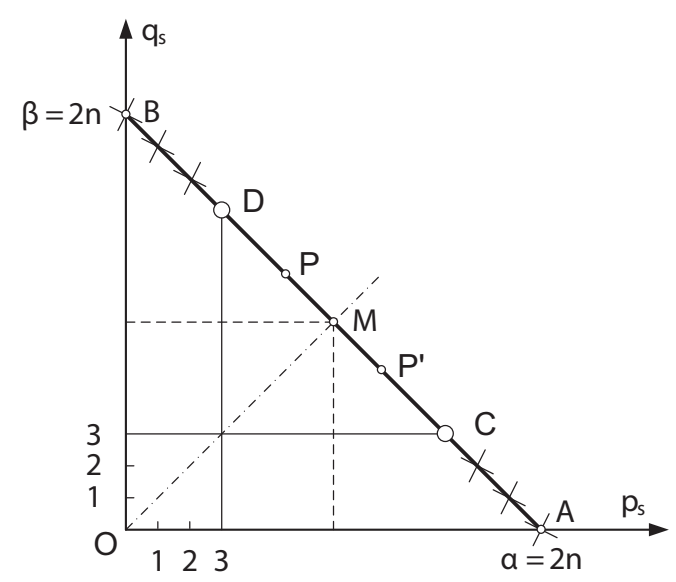

Figure 1: Diagram showing the interval CD on which lie the arranged pairs $\left(p_{s}, q_{s}\right)$ which satisfy the relationship $p_{s}+q_{s}=2 n, n>2$. To avoid repeated pairs, we work only on the part DM, where M denotes the common mid-point of the straight line segments $\mathrm{AB}$ and $\mathrm{CD}$.

A better representation, especially for various small numbers is detailed in Table 1. In full agreement with the immediately above, we observe that the middle $\mathrm{M}$ appears to be among the candidate pairs only when the number $n$ is odd, i.e. for the even numbers: $6,10,14, \ldots, 50$, and so on. But if we consider the middle $\mathrm{M}$ of segment $\mathrm{CD}$, each pair $\mathrm{P}\left(p_{s}, q_{s}\right)$ has a corresponding equivalent pair being represented by the symmetric point $\mathrm{P}^{\prime}\left(q_{s}, p_{s}\right)$ of $\mathrm{P}$ with respect to M. If we want to exclude the repetition of a pair $\left(p_{s}, q_{s}\right)$, then we can restrict our attention only to half of CD, e.g. the segment DM. Concerning the point $\mathrm{M}$, it satisfies the relation $p_{s}+q_{s}=2 n$ only when the number $n$ is odd.

Theorem 3. Suppose the point $P$ in Figure 1 starts from point $D$ and 


$2 \mathrm{v}+1 \mathrm{v}$
\begin{tabular}{|r|r|r|l|l|l|l|l|l|l|l|l|l|l|l|}
\hline $\mathbf{2 5}$ & $\mathbf{1 2}$ & 28 & 30 & 32 & 34 & 36 & 38 & 40 & 42 & 44 & 46 & 48 & 50 & \\
\hline $\mathbf{2 3}$ & $\mathbf{1 1}$ & 26 & 28 & 30 & 32 & 34 & 36 & 38 & 40 & 42 & 44 & 46 & 48 & \\
\hline $\mathbf{2 1}$ & $\mathbf{1 0}$ & 24 & 26 & 28 & 30 & 32 & 34 & 36 & 38 & 40 & 42 & 44 & 46 & \\
\hline $\mathbf{1 9}$ & $\mathbf{9}$ & 22 & 24 & 26 & 28 & 30 & 32 & 34 & 36 & 38 & 40 & 42 & 44 & \\
\hline $\mathbf{1 7}$ & $\mathbf{8}$ & 20 & 22 & 24 & 26 & 28 & 30 & 32 & 34 & 36 & 38 & 40 & 42 & \\
\hline $\mathbf{1 5}$ & $\mathbf{7}$ & 18 & 20 & 22 & 24 & 26 & 28 & 30 & 32 & 34 & 36 & 38 & 40 & \\
\hline $\mathbf{1 3}$ & $\mathbf{6}$ & 16 & 18 & 20 & 22 & 24 & 26 & 28 & 30 & 32 & 34 & 36 & 38 & \\
\hline $\mathbf{1 1}$ & $\mathbf{5}$ & 14 & 16 & 18 & 20 & 22 & 24 & 26 & 28 & 30 & 32 & 34 & 36 & \\
\hline $\mathbf{9}$ & $\mathbf{4}$ & 12 & 14 & 16 & 18 & 20 & 22 & 24 & 26 & 28 & 30 & 32 & 34 & \\
\hline $\mathbf{7}$ & $\mathbf{3}$ & 10 & 12 & 14 & 16 & 18 & 20 & 22 & 24 & 26 & 28 & 30 & 32 & \\
\hline $\mathbf{5}$ & $\mathbf{2}$ & 8 & 10 & 12 & 14 & 16 & 18 & 20 & 22 & 24 & 26 & 28 & 30 & \\
\hline $\mathbf{3}$ & $\mathbf{1}$ & 6 & 8 & 10 & 12 & 14 & 16 & 18 & 20 & 22 & 24 & 26 & 28 & \\
\hline & $\mathbf{0}$ & $\mathbf{1}$ & $\mathbf{2}$ & $\mathbf{3}$ & $\mathbf{4}$ & $\mathbf{5}$ & $\mathbf{6}$ & $\mathbf{7}$ & $\mathbf{8}$ & $\mathbf{9}$ & $\mathbf{1 0}$ & $\mathbf{1 1}$ & $\mathbf{1 2}$ & $\mathrm{V}$ \\
\hline & & $\mathbf{3}$ & $\mathbf{5}$ & $\mathbf{7}$ & $\mathbf{9}$ & $\mathbf{1 1}$ & $\mathbf{1 3}$ & $\mathbf{1 5}$ & $\mathbf{1 7}$ & $\mathbf{1 9}$ & $\mathbf{2 1}$ & $\mathbf{2 3}$ & $\mathbf{2 5}$ & $2 \mathrm{v}+1$ \\
\hline
\end{tabular}

Table 1: Representative way of decomposing even numbers in a sum of two odd ones. Every even integer has been put in the form $2 n \equiv 2(\nu+2)$, and is produced by the sum of red odd numbers $(2 \nu+1)$ of the horizontal axis and the corresponding red values in the vertical axis. The colors displayed in green, blue and magenta correspond to the even numbers of the form $6 n, 6 n+2$ and $6 n-2$, respectively $(n \in \mathbb{N})$.

moves towards $M$ traversing all distinct pairs $\left(p_{s}, q_{s}\right)$ on the straight segment $D M$ with $p_{s} \leq q_{s}$. In this movement, two sets $A$ and $B$ of odd natural numbers are created, of which the first $(A)$ is formed by the values of $p_{s}$ while the second (B) by the values of $q_{s}$. Both sets have the same cardinality: $n_{s}=\lfloor(n-1) / 2\rfloor$, which equals the number of distinct pairs that satisfy the relationship $p_{s}+q_{s}=$ $2 n$. Assuming that the number of prime numbers in sets $A$ and $B$ are $\alpha_{1}$ and $\beta_{1}$, respectively, and that the distribution of primes along the arithmetic line is completely random, the number of pairs of primes $(p, q)$ that satisfy Eq(1), is approximated by:

$$
n_{p}=\left(\alpha_{1} \beta_{1}\right) / n_{s}
$$

Proof. According to the theory of probability, in each pair $\left(p_{s}, q_{s}\right)$ selected from the set of $n_{s}=\lfloor(n-1) / 2\rfloor$ elements, there are four possible events as shown in Table 2.

If we select a random pair $\left(p_{s}, q_{s}\right)$, where $p_{s} \in A \wedge q_{s} \in B$, the probability $p_{s}$ be prime is $P_{A}=\alpha_{1} / n_{s}$, while the probability $q_{s}$ be prime is $P_{B}=\beta_{1} / n_{s}$. Since the first event $\left(p_{s}=\right.$ prime) is entirely independent on the second event ( $q_{s}=$ prime), the probability $P_{\text {prime }}$ of the intersection of these two independent 


\begin{tabular}{ccc}
\hline $\begin{array}{c}\text { Column A } \\
\text { Prime }\end{array}$ & Column B & Number of pairs \\
Prime & Composite & $n_{p p}=\left(\alpha_{1} \beta_{1}\right) / n_{s}$ \\
Composite & Prime & $n_{p c}=\alpha_{1}\left(n_{s}-\beta_{1}\right) / n_{s}$ \\
Composite & Composite & $n_{c p}=\left(n_{s}-\alpha_{1}\right) \beta_{1} / n_{s}$ \\
Sum of pairs & & $n_{c c}\left(n_{s}-\alpha_{1}\right)\left(n_{s}-\beta_{1}\right) / n_{s}$ \\
& & $n_{s}$ \\
\hline
\end{tabular}

Table 2: All possible combinations for the formation of a pair $\left(p_{s}, q_{s}\right)$ from the columns A and B.

events equals to the product of their probabilities, that is:

$$
P_{\text {prime }}=P_{A} \cdot P_{B}=\left(\alpha_{1} \beta_{1}\right) / n_{s}^{2}
$$

Since the probability $P_{\text {prime }}$ on the sample of $n_{s}$ pairs is known, the number $n_{p}$ of pairs of prime numbers $(p, q)$ will be also known and will be approximated by the relationship:

$$
n_{p}=P_{\text {prime }} \cdot n_{s}=\left(\alpha_{1} \beta_{1}\right) / n_{s}
$$

The relationship (15) completes the proof of Theorem-3.

It is noted that, generally, probabilistic analyzes are conducted under conditions of uncertainty. When talking about probability, we refer to the realization of one event in relation to other possible events. Obviously, the possibility to verify Goldbach's conjecture on the number of $n_{p}$ pairs, belongs to the first category (13a).

Remark. The distribution of prime numbers on the numerical line is not accidental (stochastic) but strictly predetermined. It is therefore a purely deterministic phenomenon. The prime numbers are in a predetermined position, waiting to be discovered.

Today, we know the number and distribution of prime numbers for a very large number of natural numbers. Therefore, we know the number of primes, both in column $\mathrm{A}\left(\alpha_{1}\right)$ and in column $\mathrm{B}\left(\beta_{1}\right)$, arranged in $n_{s}$ pairs in which the even numbers are decomposed on the basis of equations (9) and (10). So, we are able to rigorously examine whether the predetermined distribution of prime numbers is such as to ensure the validity of probabilistic relationship (13a), "prime-to-prime" in a representative sample where the number of pairs $n_{s}$ is small. 
As an example, Table 3 represents the way in which we decompose a triple (triad) of even numbers $(6 n-2,6 n, 6 n+2)$, where $n=15$. To facilitate discrimination of pairs "prime-to-prime", we encode the composite numbers with gray color, the odd (prime numbers) of the form $6 \lambda-1$ with turquoise color, the odd (prime numbers) of the form $6 \lambda+1$ with tile color, and finally the prime number 3 with yellow color. Since even numbers of the form $6 n$ are produced on the basis of two equations, i.e. (9b) and (9c), while the even numbers of either the form $6 n-2$ or $6 n+2$ are produced by only one equation [(9a) or (9d), respectively], an asymmetry appears in the number of ordered pairs that verify (13a), as shown in Table 3.

Figure 2 shows, with a remarkable correlation, the expected 'coincidence' of the curve which represents the number of cases that verify Goldbach's conjecture, only for the even numbers of the form $6 n$ (on one hand) and the sum of cases for the even numbers in the form $6 n-2$ and $6 n+2$ (on the other hand).

Figure 3 shows, separately for each form of the triad of even numbers $(6 n, 6 n-2,6 n+2)$, the number of pairs (dyads) of prime numbers that verify Goldbach's conjecture. As previously observed, even in this case the anticipated 'coincidence' by equations (9a) and (9d) occurs.

To remove the above asymmetry, due to the four relations (9), which in turn reflect the three types of even numbers, we transform (13a) by introducing three weights whose sum is equal to 3 (the weight of $6 n$ is twice the others), and therefore adapt in more detail as follows:

$$
\begin{array}{rrrlrl}
\text { For } & 6 n-2: & & n_{p p} & =3 / 4\left(\alpha_{1} \beta_{1} / n_{s}\right) \\
\text { For } & 6 n: & n_{p p} & =3 / 2\left(\alpha_{1} \beta_{1} / n_{s}\right) \\
\text { For } & 6 n+2: & n_{p p} & =3 / 4\left(\alpha_{1} \beta_{1} / n_{s}\right)
\end{array}
$$

Therefore, if we assume that Goldbach's conjecture is not verified for some even numbers, these cases should be essentially searched within the even numbers of the form $6 n-2$ and $6 n+2$, which have the smallest values of verification, those of $\mathrm{Eq}(16 \mathrm{a})$ and $\mathrm{Eq}(16 \mathrm{c})$.

\section{The Distribution of Prime Numbers}

It is known, by virtue of the Prime Number Theorem (PNT) [see Appendix] that, as we move to larger numbers $N$, the density of primes $1 / \log N$ gradually decreases. In the following, the symbol $\log x[$ or $\log (x)]$ is equivalent to the 


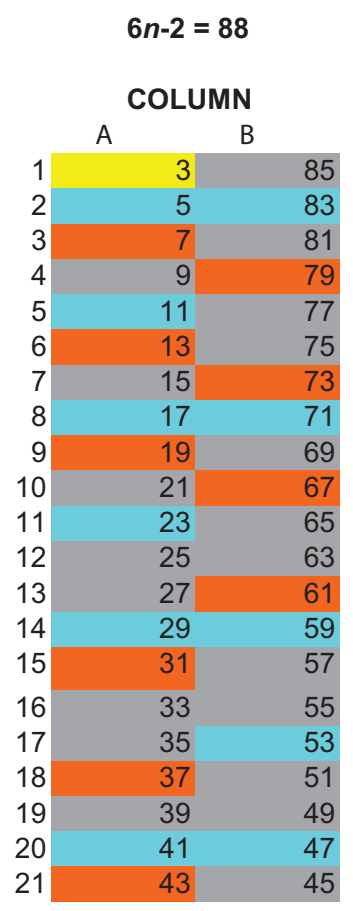

Sum of pairs that fulfill Goldbach conjecture: 4

Sum of Pairs: 21 [Equation (9a)]

\section{Composite number}

Odd prime in the form $(6 \lambda-1)$

Odd prime in the form $(6 \lambda+1)$

Prime number 3

$$
6 n=90
$$

COLUMN

\begin{tabular}{|c|c|c|}
\hline \multicolumn{3}{|c|}{$\begin{array}{ll}A & B\end{array}$} \\
\hline 1 & 3 & 87 \\
\hline 2 & 5 & 85 \\
\hline 3 & 7 & 83 \\
\hline 4 & 9 & 81 \\
\hline 5 & 11 & 79 \\
\hline 6 & 13 & 77 \\
\hline 7 & 15 & 75 \\
\hline 8 & 17 & 73 \\
\hline 9 & 19 & 71 \\
\hline 10 & 21 & 69 \\
\hline 11 & 23 & 67 \\
\hline 12 & 25 & 65 \\
\hline 13 & 27 & 63 \\
\hline 14 & 29 & 61 \\
\hline 15 & 31 & 59 \\
\hline 16 & 33 & 57 \\
\hline 17 & 35 & 55 \\
\hline 18 & 37 & 53 \\
\hline 19 & 39 & 51 \\
\hline 20 & 41 & 49 \\
\hline 21 & 43 & 47 \\
\hline 22 & 45 & 45 \\
\hline
\end{tabular}

Sum of pairs that fulfill Goldbach conjecture: 9

Sum of Pairs: 22 [Equations (9b) and (9c)]

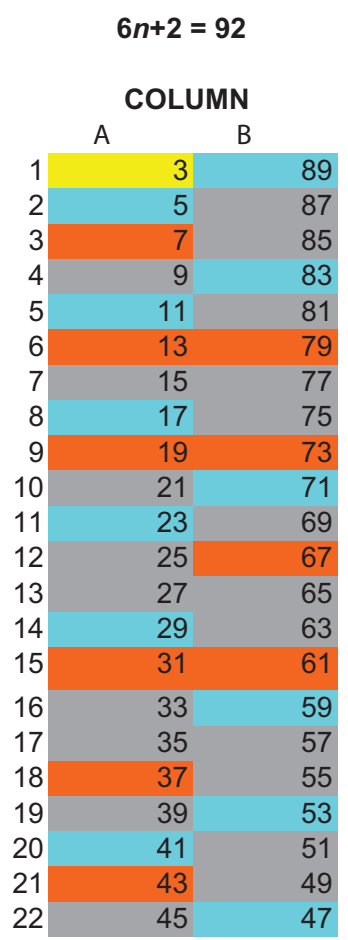

Sum of pairs that fulfill Goldbach conjecture: $\mathbf{4}$

Sum of Pairs: 22 [Equation (9d)]

\section{(Sum of pairs fulfilling Golbach's conjecture for the entire triad: $4+9+4=17$ )}

Table 3: Example for the decomposition of a triad of numbers $(6 n-$ $2,6 n, 6 n+2)$, for $n=15$, in sum of two odd numbers.

symbol $\ln x$ [or $\ln (x)]$ corresponding to the Neperian (natural) logarithm of base $e$.

Here we should clarify that, despite the gradual decrease in the density of the primes (see percentage in the fifth column of Table 4), the odd numbers 


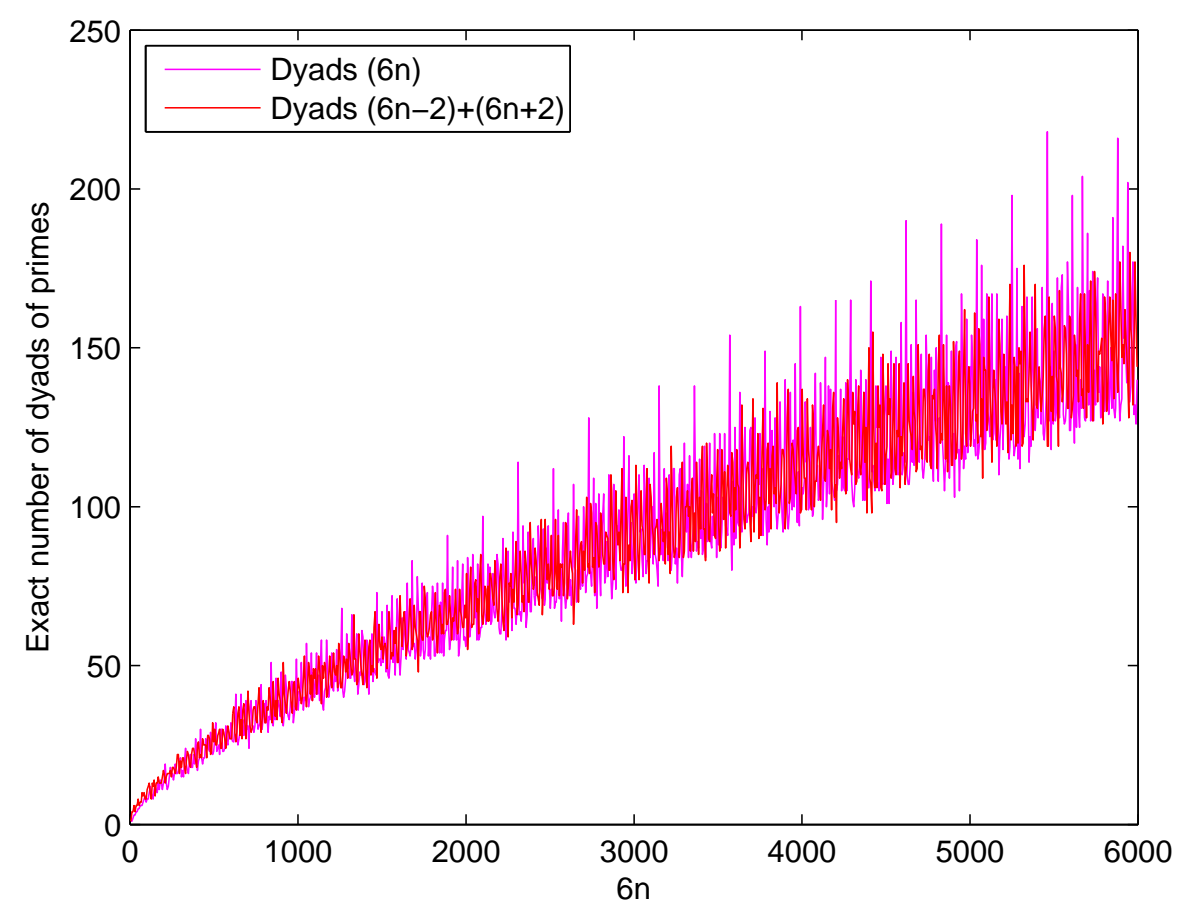

Figure 2: Number of pairs (dyads) of prime numbers that fulfill Goldbach's conjecture for the lowest 6000 numbers, in two characteristic categories [magenta line: dyads $6 n$, red line: dyads $(6 n-2)$ and $(6 n+2)]$.

starting from the number 3 (from which we start the column A) until the even number of $6 n+2=92$ (see right column of Table 3 ) is 44 . Within these 44 odd numbers, 23 of primes are contained. Thus the percentage of primes in the ordered pairs of odd number in which the even number 92 is analyzed will be $23 \times 44 / 100=52.3 \%$. This implies that in any distribution of prime and composite numbers, in the ordered in pairs in which the even numbers between 6 and 92 are analyzed, the value of any prime-to-prime event will be larger than 1. This means that Goldbach's conjecture is a priori verified for the even numbers with $n \leq 15$.

Based on the default deterministic creation of ordered pairs, to ensure the sum of all dyads be $6 n-2$, and given the distribution of prime numbers in between 3 and $6 \times 15-2=88$, the number $n_{p p}$ of verifications is illustrated in Table 4, where we present the data $\alpha_{1}, \beta_{1}$ and $n_{s}$, involved in (16a) to 


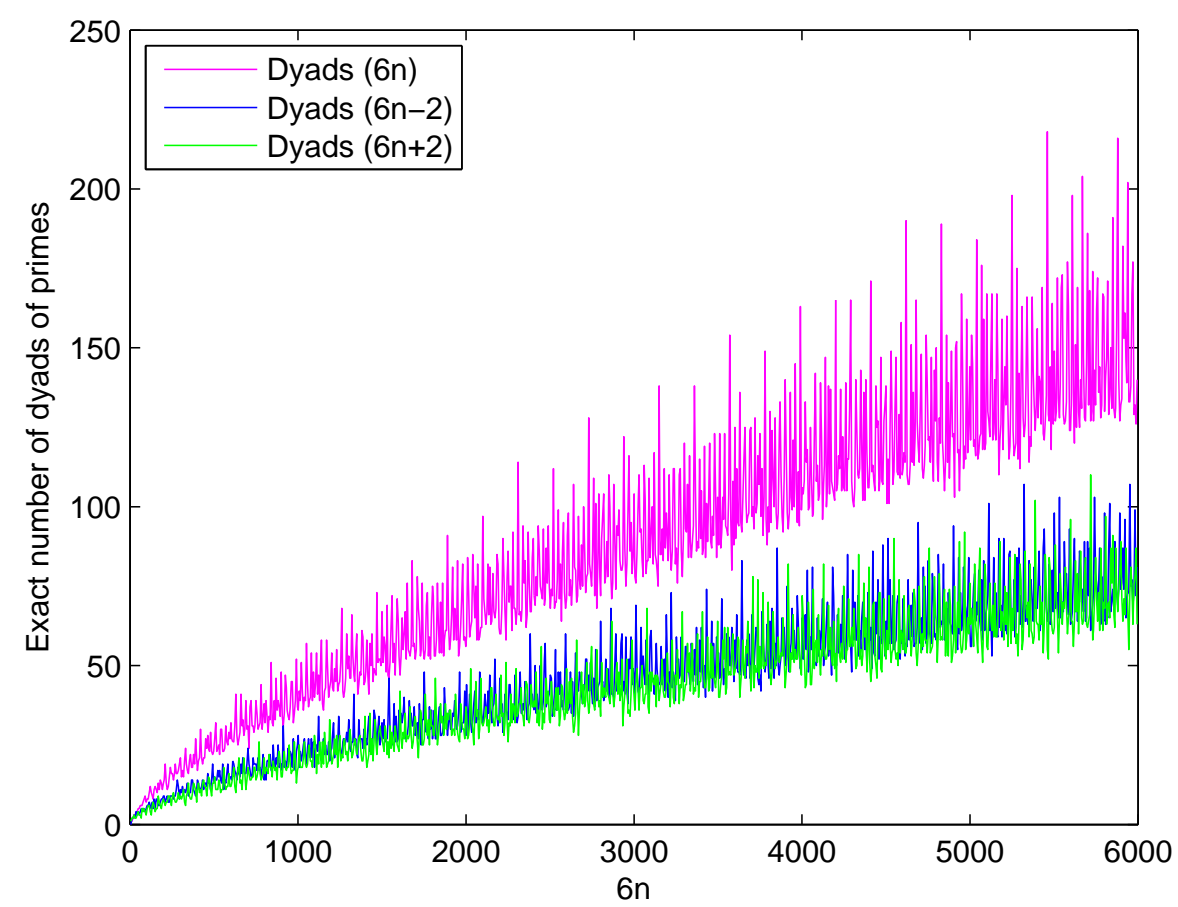

Figure 3: Number of dyads of prime numbers that fulfill Goldbach's conjecture for the smallest 6000 numbers, in three characteristic categories according to the cell $(6 n-2,6 n, 6 n+2)$.

calculate $n_{p p}$, for even numbers of the form $6 n-2$ with $n$ varying from 2 to 15. A first observation arising from the data of Table 4 is that the density and distribution of primes in that interval is such as to ensure in a great approach, the coincidence of the values derived from (16a) with actual checks.

The density and distribution of prime numbers also ensure that $\alpha_{1}$ and $\beta_{1}$ increase in such a way that the product $\left(\alpha_{1} \beta_{1}\right)$ increases at a faster rate than the rate at which the number $n_{s}$ of ordered pairs increases. Direct result of the distribution of prime numbers is that as we move to larger even numbers, the verification of Goldbach's conjecture constantly increases, and is increasingly removed far away from the value ' 1 ' required by the conjecture.

Therefore, besides the percentage (\%) which, in the test sample, ensures a priori verification of Goldbach conjecture, it is also the distribution of prime numbers, and more especially the distribution of the primes, which ensures the 


\begin{tabular}{|l|l|l|l|l|l|l|l|}
\hline$n$ & $6 n-2$ & $\alpha_{1}$ & $\beta_{1}$ & $\begin{array}{l}\text { Percentage } \\
(\%)\end{array}$ & $n_{s}$ & $\begin{array}{l}\text { Number } \\
\text { of verifica- } \\
\text { tions } n_{p p} \\
\text { according } \\
\text { to Eq(16a) }\end{array}$ & True $n_{p p}$ \\
\hline 2 & 10 & 2 & 2 & 100 & 2 & 1.5 & 2 \\
3 & 16 & 3 & 2 & 80 & 3 & 1.5 & 2 \\
4 & 22 & 4 & 4 & 80 & 5 & 2.4 & 3 \\
5 & 28 & 5 & 3 & 67 & 6 & 1.9 & 2 \\
6 & 34 & 6 & 5 & 69 & 8 & 2.8 & 4 \\
7 & 40 & 7 & 4 & 61 & 9 & 2.3 & 3 \\
8 & 46 & 8 & 6 & 64 & 11 & 3.3 & 4 \\
9 & 52 & 8 & 6 & 58 & 12 & 3.0 & 3 \\
10 & 58 & 9 & 7 & 57 & 14 & 3.4 & 4 \\
11 & 64 & 10 & 7 & 57 & 15 & 3.5 & 5 \\
12 & 70 & 10 & 8 & 53 & 17 & 3.5 & 5 \\
13 & 76 & 11 & 9 & 55 & 18 & 4.1 & 5 \\
14 & 82 & 12 & 10 & 55 & 20 & 4.5 & 5 \\
15 & 88 & 13 & 9 & 52 & 21 & 4.2 & 4 \\
\hline
\end{tabular}

Table 4: Estimation of the number of verifications $n_{p p}$ concerning Goldbach's conjecture based on Eq(16a) compared with true values.

appropriate values in $\alpha_{1}$ and $\beta_{1}$ so that the results derived from (16) to be in a much closed agreement with the actual verification. This fact urges us to investigate whether the distribution of prime numbers, in a larger and more representative sample of even numbers, where the percentage (\%) of the primes with its continuous reduction, goes down below 50\%, shall ensure, by itself, the values for $\alpha_{1}$ and $\beta_{1}$ which are also in agreement with the actual verifications.

Due to the reduced space, in Table 5 the following quantities are randomly recorded:

-the values of $\alpha_{1}$ and $\beta_{1}$,

-the number of columns $n_{s}$

-and the verifications $n_{p p}$ based on equations (16),

for several values of the even numbers in the form $6 n-2,6 n$ and $6 n+2$ for $n=20$ until $n=2000$, which correspond to the even numbers $(118,120,122)$ until (11998, 12000, 12002).

The even number 12002 includes 6001 odd numbers and 1437 primes. Therefore, the percentage (in \%) of the primes included in the 6001 odd numbers by 
which we create the ordered pairs, will be $1437 / 6001 \times 100=23.9 \%$. Obviously, this percentage does not ensure the a-priori verification of Goldbach's conjecture.

In the sequence, Table 6 includes both the actual verifications of Goldbach's conjecture, in red, and the verifications based on formula (16); the latter are due to the distribution of prime numbers $\left(\alpha_{1}, \beta_{1}\right)$, in columns $\mathrm{A}$ and $\mathrm{B}$, in which each even number is decomposed according to $\mathrm{Eq}(9)$ and $\mathrm{Eq}(10)$, in blue (already included in Table 5). From the results in Table 6, one can notice that the distribution of prime numbers is enough to ensure the values of the verification under $\mathrm{Eq}(16)$ to be in close accordance with the reality, as clearly shown in Figure 4 (for these particular results only).

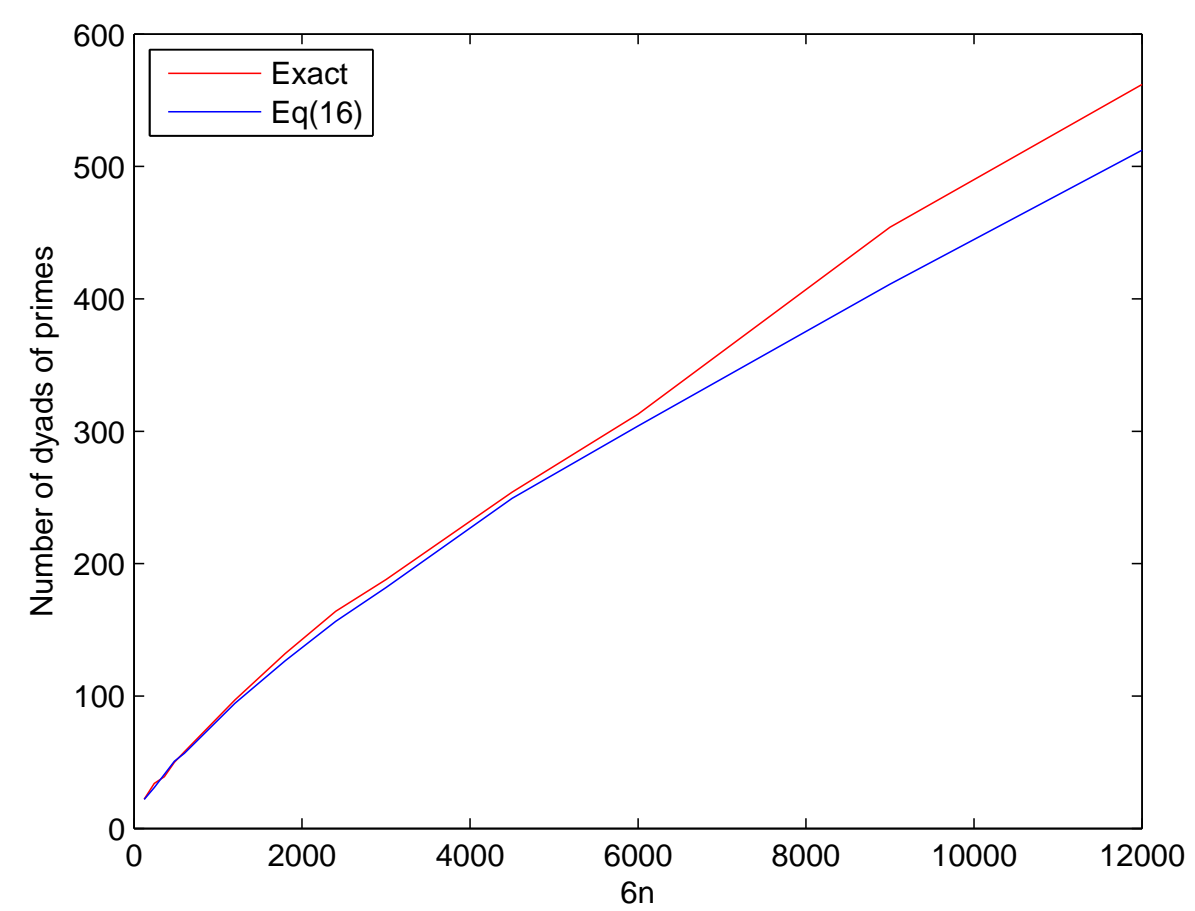

Figure 4: Number of pairs (dyads) of primes that fulfill Goldbach Conjecture for the smallest 12000 numbers (the red line represents the real number of dyads of primes that fulfill Goldbach Conjecture, while the blue line corresponds to the $\mathrm{Eq}(16))$. The graph is based on Table 6 only. 


\begin{tabular}{|c|c|c|c|c|}
\hline For $n=20$ & $a_{1}$ & $\beta_{1}$ & $n_{s}$ & $n_{p p}[\operatorname{Eqs}(16)]$ \\
\hline $6 n-2=118$ & 16 & 14 & 29 & $3 / 4 a_{1} \beta_{1} / n_{s}=3 / 4^{*} 16^{*} 14 / 29=5.8:$ Eq 16(a) \\
\hline $6 n=120$ & 16 & 13 & 29 & $3 / 2 a_{1} \beta_{1} / n_{s}=3 / 4 * 16^{*} 13 / 29=10.7:$ Eq $16(b)$ \\
\hline \multirow[t]{2}{*}{$6 n+2=122$} & 17 & 13 & 30 & $3 / 4 a_{1} \beta_{1} / n_{s}=3 / 4^{*} 17^{*} 13 / 30=5.5:$ Eq 16(c) \\
\hline & & & & Sum of triad $=21.9$ \\
\hline For $n=40$ & $a_{1}$ & $\beta_{1}$ & $n_{s}$ & \\
\hline $6 n-2=238$ & 29 & 21 & 59 & $3 / 4 a_{1} \beta_{1} / n_{s}=3 / 4^{*} 29^{*} 21 / 59=7.7$ \\
\hline $6 n=240$ & 29 & 21 & 59 & $3 / 2 \alpha_{1} \beta_{1} / n_{s}=3 / 2 * 29 * 21 / 59=15.5$ \\
\hline \multirow[t]{2}{*}{$6 n+2=242$} & 29 & 21 & 60 & $3 / 4 \alpha_{1} \beta_{1} / n_{s}=3 / 4^{*} 29^{*} 21 / 60=7.6$ \\
\hline & & & & Sum of triad $=30.8$ \\
\hline For $n=60$ & $a_{1}$ & $\beta_{1}$ & $n_{s}$ & \\
\hline $6 n-2=358$ & 40 & 31 & 89 & $3 / 4 \alpha_{1} \beta_{1} / n_{s}=3 / 4 * 40 * 31 / 89=10.4$ \\
\hline $6 n=360$ & 40 & 30 & 89 & $3 / 2 \alpha_{1} \beta_{1} / n_{s}=3 / 2 * 40 * 30 / 89=20.2$ \\
\hline \multirow[t]{2}{*}{$6 n+2=362$} & 41 & 30 & 90 & $3 / 4 a_{1} \beta_{1} / n_{s}=3 / 4^{*} 41^{*} 30 / 90=10.2$ \\
\hline & & & & Sum of triad $=40.8$ \\
\hline For $n=80$ & $a_{1}$ & $\beta_{1}$ & $n_{s}$ & \\
\hline $6 n-2=478$ & 51 & 40 & 119 & $3 / 4 a_{1} \beta_{1} / n_{s}=3 / 4^{*} 51^{*} 40 / 119=12.9$ \\
\hline $6 n=480$ & 51 & 39 & 119 & $3 / 2 \alpha_{1} \beta_{1} / n_{s}=3 / 2 * 51 * 39 / 119=25.1$ \\
\hline \multirow[t]{2}{*}{$6 n+2=482$} & 52 & 39 & 120 & $3 / 4 a_{1} \beta_{1} / n_{s}=3 / 4 * 54^{*} 41 / 120=12.7$ \\
\hline & & & & Sum of triad $\quad=50.7$ \\
\hline For $n=100$ & $a_{1}$ & $\beta_{1}$ & $n_{s}$ & \\
\hline $6 n-2=598$ & 61 & 46 & 149 & $3 / 4 a_{1} \beta_{1} / n_{s}=3 / 4^{*} 61 * 46 / 149=14.1$ \\
\hline $6 n=600$ & 61 & 46 & 149 & $3 / 2 \alpha_{1} \beta_{1} / n_{s}=3 / 2^{*} 61^{*} 46 / 149=28.2$ \\
\hline \multirow[t]{2}{*}{$6 n+2=602$} & 61 & 47 & 150 & $3 / 4 a_{1} \beta_{1} / n_{s}=3 / 4 * 61 * 47 / 150=14.3$ \\
\hline & & & & Sum of triad $\quad=56.6$ \\
\hline For $n=200$ & $a_{1}$ & $\beta_{1}$ & $n_{s}$ & \\
\hline $6 n-2=1198$ & 108 & 88 & 299 & $3 / 4 a_{1} \beta_{1} / n_{s}=3 / 4^{*} 108^{*} 88 / 299=23.8$ \\
\hline $6 n=1200$ & 108 & 87 & 299 & $3 / 2 a_{1} \beta_{1} / n_{s}=3 / 2^{*} 108^{*} 87 / 299=47.1$ \\
\hline \multirow[t]{2}{*}{$6 n+2=1202$} & 109 & 87 & 300 & $3 / 4 a_{1} \beta_{1} / n_{s}=3 / 4^{*} 109^{*} 87 / 300=23.7$ \\
\hline & & & & Sum of triad $=94.6$ \\
\hline For $n=300$ & $a_{1}$ & $\beta_{1}$ & $n_{s}$ & \\
\hline $6 n-2=1798$ & 153 & 124 & 449 & $3 / 4 a_{1} \beta_{1} / n_{s}=3 / 4^{*} 153^{*} 124 / 449=31.7$ \\
\hline $6 n=1800$ & 153 & 124 & 449 & $3 / 2 a_{1} \beta_{1} / n_{s}=3 / 2 * 153^{*} 124 / 449=63.4$ \\
\hline \multirow[t]{2}{*}{$6 n+2=1802$} & 153 & 124 & 450 & $3 / 4 a_{1} \beta_{1} / n_{s}=3 / 4 * 153^{*} 124 / 450=31.6$ \\
\hline & & & & Sum of triad $\quad=126.7$ \\
\hline For $n=400$ & $a_{1}$ & $\beta_{1}$ & $n_{s}$ & \\
\hline $6 n-2=2398$ & 195 & 160 & 599 & $3 / 4 a_{1} \beta_{1} / n_{s}=3 / 4^{*} 195^{*} 160 / 599=39.0$ \\
\hline $6 n=2400$ & 195 & 160 & 599 & $3 / 2 \alpha_{1} \beta_{1} / n_{s}=3 / 2^{*} 195^{*} 160 / 599=78.1$ \\
\hline \multirow[t]{2}{*}{$6 n+2=2402$} & 196 & 161 & 600 & $3 / 4 a_{1} \beta_{1} / n_{s}=3 / 4^{*} 196^{*} 161 / 600=39.4$ \\
\hline & & & & Sum of triad $\quad=156.5$ \\
\hline For $n=500$ & $a_{1}$ & $\beta_{1}$ & $\boldsymbol{n}_{\mathrm{s}}$ & \\
\hline $6 n-2=2998$ & 238 & 191 & 749 & $3 / 4 \alpha_{1} \beta_{1} / n_{s}=3 / 4^{*} 238^{*} 191 / 749=45.5$ \\
\hline $6 n=3000$ & 238 & 190 & 749 & $3 / 2 \alpha_{1} \beta_{1} / n_{s}=3 / 2^{*} 238^{*} 191 / 749=91.0$ \\
\hline \multirow[t]{2}{*}{$6 n+2=3002$} & 238 & 191 & 750 & $3 / 4 \alpha_{1} \beta_{1} / n_{s}=3 / 4^{*} 238^{*} 191 / 450=45.4$ \\
\hline & & & & Sum of triad $\quad=181.9$ \\
\hline For $n=750$ & $a_{1}$ & $\beta_{1}$ & $n_{s}$ & \\
\hline $6 n-2=4498$ & 333 & 276 & 1124 & $3 / 4 a_{1} \beta_{1} / n_{s}=3 / 4^{*} 333^{*} 276 / 1124=61.3$ \\
\hline $6 n=4500$ & 333 & 276 & 1124 & $3 / 2 \alpha_{1} \beta_{1} / n_{s}=3 / 2^{*} 333^{*} 276 / 1124=126.6$ \\
\hline \multirow[t]{2}{*}{$6 n+2=4502$} & 334 & 276 & 1125 & $3 / 4 a_{1} \beta_{1} / n_{s}=3 / 4^{*} 334^{*} 276 / 1122=61.3$ \\
\hline & & & & Sum of triad $\quad=249.2$ \\
\hline For $n=1000$ & $a_{1}$ & $\beta_{1}$ & $n_{s}$ & \\
\hline $6 n-2=5998$ & 429 & 354 & 1499 & $3 / 4 \alpha_{1} \beta_{1} / n_{s}=3 / 4 * 429 * 354 / 1499=76$ \\
\hline $6 n=6000$ & 429 & 354 & 1499 & $3 / 2 \alpha_{1} \beta_{1} / n_{s}=3 / 2 * 429 * 354 / 1499=152$ \\
\hline \multirow[t]{2}{*}{$6 n+2=6002$} & 430 & 354 & 1500 & $3 / 4 \alpha_{1} \beta_{1} / n_{s}=3 / 4 * 430 * 354 / 1500=76$ \\
\hline & & & & Sum of triad $=304$ \\
\hline For $n=1500$ & $a_{1}$ & $\beta_{1}$ & $n_{s}$ & \\
\hline $6 n-2=8998$ & 609 & 506 & 2249 & $3 / 4 a_{1} \beta_{1} / n_{s}=3 / 4^{*} 609^{*} 506 / 2249=102.8$ \\
\hline $6 n=9000$ & 609 & 506 & 2249 & $3 / 2 \alpha_{1} \beta_{1} / n_{\mathrm{s}}=3 / 2 * 609 * 506 / 2249=205.5$ \\
\hline \multirow[t]{2}{*}{$6 n+2=9002$} & 609 & 507 & 2250 & $3 / 4 a_{1} \beta_{1} / n_{s}=3 / 4^{*} 609^{*} 507 / 2250=102.7$ \\
\hline & & & & Sum of triad $\quad=411$ \\
\hline For $n=2000$ & $a_{1}$ & $\beta_{1}$ & $n_{s}$ & \\
\hline $6 n-2=11998$ & 782 & 655 & 2999 & $3 / 4 \alpha_{1} \beta_{1} / n_{s}=3 / 4 * 782^{*} 655 / 2999=128.1$ \\
\hline $6 n=12000$ & 782 & 655 & 2999 & $3 / 2 \alpha_{1} \beta_{1} / n_{s}=3 / 2 * 782^{*} 655 / 2999=256.2$ \\
\hline \multirow[t]{2}{*}{$6 n+2=12002$} & 782 & 655 & 3000 & $3 / 4 a_{1} \beta_{1} / n_{s}=3 / 4^{*} 782^{*} 655 / 3000=128.0$ \\
\hline & & & & Sum of triad $\quad=512.3$ \\
\hline
\end{tabular}

Table 5: Estimation of the number of verifications $n_{p p}$ of Goldbach's conjecture based on relations (16) for several values of even numbers in the form $6 n-2,6 n$, and $6 n+2$. 


\begin{tabular}{|c|c|c|c|c|}
\hline n & \multicolumn{4}{|c|}{$n=\mathbf{2 0}$} \\
\hline Type of even number & $6 n-2$ & $6 n$ & $6 n+2$ & Sum \\
\hline Even number & 118 & 120 & 122 & \\
\hline Verifications of GC (Facts) & 6 & 12 & 4 & 22 \\
\hline Verifications using Eq(16) & 5.8 & 10.7 & 5.5 & 21.9 \\
\hline $\mathrm{n}$ & \multicolumn{4}{|c|}{$n=40$} \\
\hline Type of even number & $6 n-2$ & $6 n$ & $6 n+2$ & Sum \\
\hline Even number & 238 & 240 & 242 & \\
\hline Verifications of GC (Facts) & 9 & 18 & 7 & 34 \\
\hline Verifications using Eq(16) & 7.7 & 15.5 & 7.6 & 30.8 \\
\hline n & \multicolumn{4}{|c|}{$n=60$} \\
\hline Type of even number & $6 n-2$ & $6 n$ & $6 n+2$ & Sum \\
\hline Even number & 358 & 360 & 362 & \\
\hline Verifications of GC (Facts) & 10 & 22 & 7 & 39 \\
\hline Verifications using Eq(16) & 10.4 & 20.2 & 10.2 & 40.8 \\
\hline $\mathrm{n}$ & \multicolumn{4}{|c|}{$n=80$} \\
\hline Type of even number & $6 n-2$ & $6 n$ & $6 n+2$ & Sum \\
\hline Even number & 478 & 480 & 482 & \\
\hline Verifications of GC (Facts) & 11 & 29 & 10 & 50 \\
\hline Verifications using Eq(16) & 12.9 & 25.1 & 12.7 & 50.7 \\
\hline n & \multicolumn{4}{|c|}{$n=100$} \\
\hline Type of even number & $6 n-2$ & $6 n$ & $6 n+2$ & Sum \\
\hline Even number & 598 & 600 & 602 & \\
\hline Verifications of GC (Facts) & 15 & 32 & 11 & 58 \\
\hline Verifications using Eq(16) & 14.1 & 28.2 & 14.3 & 56.8 \\
\hline $\mathrm{n}$ & \multicolumn{4}{|c|}{$n=200$} \\
\hline Type of even number & $6 n-2$ & $6 n$ & $6 n+2$ & Sum \\
\hline Even number & 1198 & 1200 & 1202 & \\
\hline Verifications of GC (Facts) & 24 & 54 & 19 & 97 \\
\hline Verifications using Eq(16) & 23.8 & 47.1 & 23.7 & 94.6 \\
\hline n & \multicolumn{4}{|c|}{$n=300$} \\
\hline Type of even number & $6 n-2$ & $6 n$ & $6 n+2$ & Sum \\
\hline Even number & 1798 & 1800 & 1802 & \\
\hline Verifications of GC (Facts) & 27 & 74 & 31 & 132 \\
\hline Verifications using Eq(16) & 31.7 & 63.4 & 31.6 & 126.7 \\
\hline $\mathrm{n}$ & \multicolumn{4}{|c|}{$n=400$} \\
\hline Type of even number & $6 n-2$ & $6 n$ & $6 n+2$ & Sum \\
\hline Even number & 2398 & 2400 & 2402 & \\
\hline Verifications of GC (Facts) & 37 & 90 & 37 & 164 \\
\hline Verifications using Eq(16) & 39.0 & 78.1 & 39.4 & 156.5 \\
\hline n & \multicolumn{4}{|c|}{$n=500$} \\
\hline Type of even number & $6 n-2$ & $6 n$ & $6 n+2$ & Sum \\
\hline Even number & 2998 & 3000 & 3002 & \\
\hline Verifications of GC (Facts) & 46 & 103 & 39 & 188 \\
\hline Verifications using Eq(16) & 45.5 & 91.0 & 45.4 & 181.9 \\
\hline n & \multicolumn{4}{|c|}{$n=750$} \\
\hline Type of even number & $6 n-2$ & $6 n$ & $6 n+2$ & Sum \\
\hline Even number & 4498 & 4500 & 4502 & \\
\hline Verifications of GC (Facts) & 64 & 138 & 52 & 254 \\
\hline Verifications using Eq(16) & 61.3 & 126.6 & 61.3 & 249.2 \\
\hline n & \multicolumn{4}{|c|}{$n=1000$} \\
\hline Type of even number & $6 n-2$ & $6 n$ & $6 n+2$ & Sum \\
\hline Even number & 5998 & 6000 & 6002 & \\
\hline Verifications of GC (Facts) & 72 & 179 & 62 & 313 \\
\hline Verifications using Eq(16) & 76.0 & 152.0 & 76.0 & 304.0 \\
\hline n & \multicolumn{4}{|c|}{$n=1500$} \\
\hline Type of even number & $6 n-2$ & $6 n$ & $6 n+2$ & Sum \\
\hline Even number & 8998 & 9000 & 9002 & \\
\hline Verifications of GC (Facts) & 101 & 243 & 110 & 454 \\
\hline Verifications using Eq(16) & 102.8 & 205.5 & 102.7 & 411.0 \\
\hline n & \multicolumn{4}{|c|}{$n=2000$} \\
\hline Type of even number & $6 n-2$ & $6 n$ & $6 n+2$ & Sum \\
\hline Even number & 11998 & 12000 & 12002 & \\
\hline Verifications of GC (Facts) & 144 & 303 & 115 & 562 \\
\hline Verifications using Eq(16) & 128.1 & 256.2 & 128.0 & 512.3 \\
\hline
\end{tabular}

Table 6: Real verifications of Goldbach's Conjecture-GC (in red colour) compared with the verifications based on $\mathrm{Eq}(16)$ (in blue colour). 
Also in this second sample of 12,000 numbers $(6 \times 2000=12000)$ the growth rate of $\alpha_{1}$ and $\beta_{1}$ is greater than the growth rate of the $n_{s}$ ordered pairs, so the number of verifications $n_{p p}$ of $\mathrm{Eq}(16)$ increases continuously and removes from the unity ("1"), as is the case with actual verifications.

Comment. Among a probabilistic experiment and the creation of ordered pairs when decomposing an even number in the sum of two odd numbers, there is a substantial difference. In a probabilistic experiment, the likelihood that one out of the four possibilities of Table 2 occurs is a matter of coincidence, or luck. The order in which the various possibilities they appear in the potential space is completely random. There is no rule which specifies the type of possibility in a particular test. Completely different is the case of an even number's decomposition in a sum of two odd numbers with the creation of ordered pairs, where the potential for each of the pairs are arranged, is strictly predetermined. Therefore the analysis of an even number in sum of two odd numbers with the creation of ordered pairs is purely deterministic.

\section{The Critical Question}

\subsection{General}

Generalization is a very important process in mathematics. We take a problem and examine its behavior in a limited area, and then we try to expand the conclusions arising from the study of this sample to larger areas. We studied the behavior of a sample of all the even numbers up to 12,000 and found that the number of pairs that verify Goldbach's conjecture, have a clear upward trend, which has a close relationship with the probabilistic equation (16).

Equation (16) imposes no restriction on the size of the sample. Not even the deterministic decomposition of an even number in odd pairs, based on equations (9) and (10), has a similar problem.

The crucial question that arises here is, whether for sufficiently large even numbers the distribution of primes in the arithmetic line continues to be such as to ensure similar behavior in deterministic ordered pairs in which sufficiently large even numbers are analyzed, to that even numbers of the relatively small sample that we previously looked, so as to legitimize to generalize to all the even numbers.

Our belief is strongly 'YES', since, as we explain below, the creation of composite numbers on the numeric (arithmetic) line, is determined by strictly defined rules, uniform for the whole crowd of natural numbers. Hence the dis- 


\begin{tabular}{|c|c|c|c|c|c|c|c|c|c|c|c|c|}
\hline$E_{3}=$ & $3 \times$ & 3 & 5 & 7 & 9 & 11 & 13 & 15 & 17 & $\ldots$ & $2 n+1$ & $\ldots$ \\
\hline$E_{5}=$ & $5 \times$ & 5 & 7 & 9 & 11 & 13 & 15 & 17 & 19 & $\ldots$ & $2 n+1$ & $\ldots$ \\
\hline$E_{7}=$ & $7 \times$ & 7 & 9 & 11 & 13 & 15 & 17 & 19 & 21 & $\ldots$ & $2 n+1$ & $\ldots$ \\
\hline$E_{9}=$ & $9 \times$ & 9 & 11 & 13 & 15 & 17 & 19 & 21 & 23 & $\ldots$ & $2 n+1$ & $\ldots$ \\
\hline$E_{11}=$ & $11 \times$ & 11 & 13 & 15 & 17 & 19 & 21 & 23 & 25 & $\ldots$ & $2 n+1$ & $\ldots$ \\
\hline$E_{13}=$ & $13 \times$ & 13 & 15 & 17 & 19 & 21 & 23 & 25 & 27 & $\ldots$ & $2 n+1$ & $\ldots$ \\
\hline
\end{tabular}

Table 7: Decomposition of Composite Numbers $(\mathrm{CN})$ in a product of two odd numbers.

tribution of prime numbers, which is formed by the relationship:

Prime numbers $=$ Natural numbers - Composite numbers has to follow the same inevitability and it is strictly prescribed. The view that the distribution of primes along the arithmetic line is random and chaotic is believed to be wrong and misleading.

\subsection{Basic Rule for the Creation of Composite Numbers}

As the prime numbers (except of 2) are odd, we will seek the prime numbers in the set of odd numbers $\mathbb{N}_{1}=\{x / x=2 n+1, n \in \mathbb{N}\}$. This set, which can be written as $\mathbb{N}_{1}=\left\{\alpha_{1}, \alpha_{2}, \alpha_{3}, \ldots \alpha_{n}, \ldots\right\}$, is a countable set equivalent to $\mathbb{N}$.

The two factors in the product $(\beta \cdot v)$ in which every odd composite is analyzed, should be odd, because only the product of odd numbers results in an odd number. Therefore, the composite numbers can be partitioned into subsets of the form:

$$
E_{i}=\{x / x=(2 n+1) \times[(2 n+1),(2 n+3), \ldots(2 n+2 k+1) \ldots]\}
$$

Such composite odd numbers are those of Table 7, where we see that the smallest of the infinite odd composite numbers generated in each row is the product of the first number in the series, by itself, i.e. $3 \times 3,5 \times 5,7 \times 7,9 \times 9$, $11 \times 11, \ldots$

In each of the above infinite series of composite odds, which are multiples of $3,5,7,9$, etc., we have the first number to be a "square" $\left(3^{2}, 5^{2}, 7^{2}, \ldots\right)$, whereas everyone else is a "rectangle". Obviously, the "squares" of prime numbers cannot be transformed into "rectangles" and will be called the "original squares". Unlike the squares of prime numbers, squares of composite numbers, such as the $9 \times 9$, can be also transformed into rectangles $3 \times 27$, which we call "abusive squares". 
Easily see that the elements of the set $E_{3}=3 \times(3,5,7, \ldots 2 n+1)$ in the first row of Table 7 , which are multiples of 3 , is a periodic 'phenomenon' in the set of integer numbers and may be derived from formula (18):

$$
E_{3}:(C N)_{3}=x=9+6 \mu=3^{2}+2 \times 3 \mu, \mu=0,1,2, \ldots
$$

For $\mu=0: x=9=3 \times 3$

For $\mu=1: x=15=3 \times 5$

For $\mu=2: x=21=3 \times 7$

For $\mu=3: x=27=3 \times 9$, e.t.c.

As a periodic phenomenon, it can also be represented as a transverse wave, as follows. In a rectangular system of axes $x \mathrm{O} y$, we identify the straight line that represents the set of natural numbers, with the axis $\mathrm{O} x$. For the sake of clarity of shape, we ignore the even numbers, and we indicate only the odd ones. If one constructs a transverse wave that starts at the number 9, of wavelength $\lambda=12$ units in the set of natural numbers $\mathbb{N}$ or $\lambda^{\prime}=12 / 2=6$ units in the set of odd numbers $\mathbb{N}_{1}$, the Composite Numbers $(C N)_{3}$ described by equation (18) coincide with the intersections of the transverse wave and the axis $\mathrm{O} x$ (zero point deviation from the axis $\mathrm{O} x$ in the direction of $y$ axis, as shown in Figure 5b). The appearance of composite numbers that are multiples of 3 on the arithmetic line is the most common of any other odd number; it divides the sum of odd pairs $\mathbb{N}_{1}$ in infinite pairs of consecutive odd numbers, starting with the square of 3 and reaching the utmost ends of the arithmetic line of Figure 5a. These successive pairs, of which, as we have explained, their first number is of the form $(6 \lambda-1)$ while the second form $(6 \lambda+1)$, will be either primes or multiple of primes $>3$. All these pairs are candidates to become the Twin Primes ${ }^{1}$, as long as none of the two numbers of the pair is crossed by a subsequent wave, thus remain to be primes, as shown in Figure 5b.

But also the elements of the set $E_{5}=5 \times(5,7,9,11,13, \ldots, 2 \kappa+1 \ldots)$ constitute a periodic 'phenomenon' in the set of odd numbers $\mathbb{N}_{1}$, starting from 25 , with a wavelength $\lambda=45-25=20$ or $\lambda^{\prime}=20 / 2=10$. Formula (19) gives the multiples of 5 that are transverse sections of the wave with the axis $\mathrm{O} x$, which apparently are composite numbers:

$$
E_{5}:(C N)_{5}=x=25+10 \mu=5^{2}+2 \times 5 \mu, \mu=0,1,2, \ldots
$$

Quite similarly, the elements of $E_{7}=7 \times(7,9,11,13,15, \ldots, 2 \kappa+1 \ldots)$ are also periodic at a frequency $14 \mu$, where $\mu \in \mathbb{N}_{1}$. The first term is 49 and the formula

\footnotetext{
${ }^{1} \mathrm{~A}$ twin prime is a prime number that differs from another prime number by two, for example the twin prime pair $(3,5)$.
} 
that gives the multiples of 7 is

$$
E_{7}:(C N)_{7}=x=49+14 \mu=7^{2}+2 \times 7 \mu, \mu=0,1,2, \ldots
$$

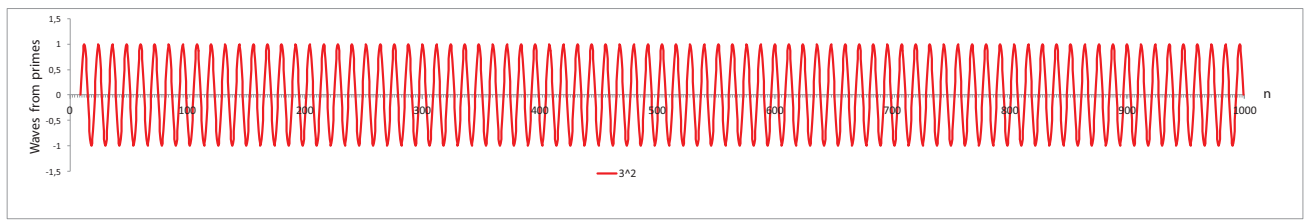

( a )

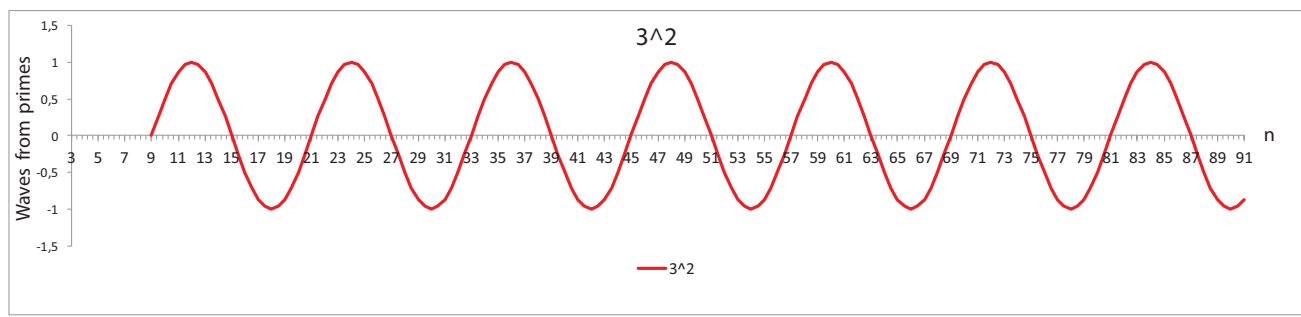

(b)

Figure 5: Formation of composite numbers starting with the square of 3 until (a) 1000 and (b) detail until 91.

Generalizing, we get a similar rationale in the calculation of the formula which gives us all the multiples of the odd number $(2 n+1)$ :

$$
E_{2 n+1}:(C N)_{2 n+1}=(2 n+1)^{2}+2(2 n+1) \mu, \mu=0,1,2, \ldots
$$

From $\mathrm{Eq}(21)$ we conclude that, as we move to increasingly odd numbers, so the composite numbers $(\mathrm{CN})$ set up by their respective primes are ever fewer because, firstly the start of a wave is shifted to the right of the numerical line, and secondly the wavelength increases.

Through a set of thousands, millions, (even for infinite remote parts of arithmetic line) waves with different wavelengths and the phase difference, some numbers of the numerical line, which is located in the heart of the package, are left untouched by all this multitude of waves. This means that there are some $N_{P}$ natural numbers which remain primes, because apparently the following relationship is satisfied:

$$
\left(N_{P}-P_{i}^{2}\right) / 2 P_{i} \mu_{i} \notin \mathbb{Z}
$$


where $P_{i}$ consecutive primes $\leq \sqrt{ } N$. That means all the following conditions are fulfilled:

$$
\left(N_{P}-25\right) / 10 \mu_{i},\left(N_{P}-49\right) / 14 \mu_{i}, \cdots,\left(N_{P}-P i^{2}\right) / 2 P_{i} \mu_{i} \notin \mathbb{Z}
$$

These numbers, being infinite and endless, as Euclid proved using the "Reductio ad absurdum" method, are the Prime Numbers (PN) that appear in Figure 6 .

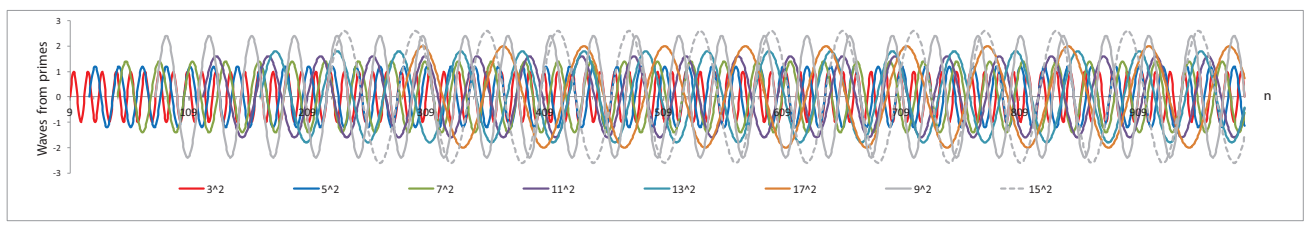

(a)

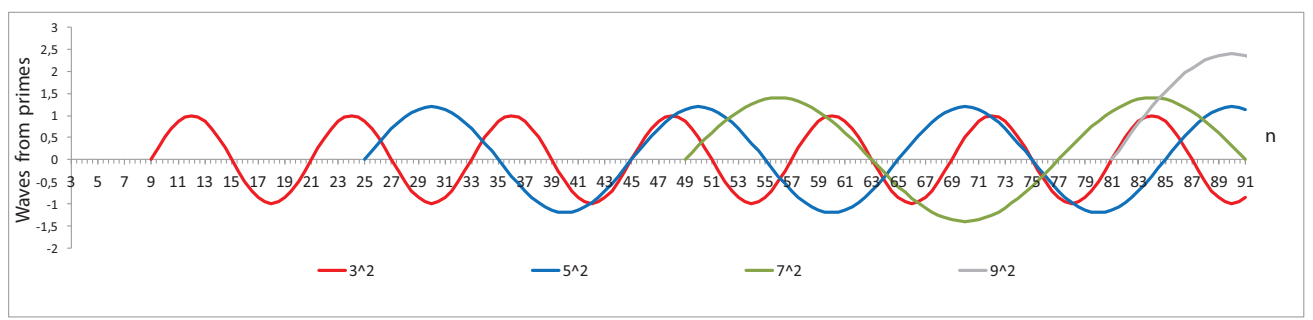

(b)

Figure 6: (a) Waves proceeding from the primes $(3,5,7,11,13,17)$ as well as the composites $(9,15)$. (b) Detail for the primes $(3,5,7)$ and the composite number 9 .

As we can see from Figure 6, only the waves starting at the squares of primes (original squares) create composite numbers. In contrast, the waves starting from composite numbers, having a growing density as we move to larger numbers, do not create new composite numbers. That's why we call them "sterile waves".

If therefore from the set $\mathbb{N}_{1}$ of odd numbers we create subsets $A_{i}$, having as first term of each successive subset the squares of all odd numbers and last term the odd number which is the next smallest square of the next odd, namely:

$$
A_{i}=\left\{x / x:(2 i+1)^{2} \leq x<(2 i+3)^{2}, x=\text { odd }\right\}
$$

such a subset will include $(2 i+3)^{2}-(2 i+1)^{2}=8(i+1)$ terms that belong to the set of natural numbers $\mathbb{N}$. Therefore, the number of terms in the set $\mathbb{N}_{1}$ 
of odd ones will be:

$$
N_{1}=8(i+1) / 2=4(i+1) \quad \text { where } \quad i \in \mathbb{N} .
$$

Due to the way of their creation (from the square of the odd $2 i+1$ up to the previous odd number than the square of $2 i+3$ ), the subsets $A_{i}$ are disjoint, and the union of all these subsets equals the set $\mathbb{N}_{1}$ of the odd numbers:

$$
A_{0} \cup A_{1} \cup A_{2} \cup A_{3} \cdots=\mathbb{N}_{1}
$$

where $($ card $=$ cardinality $)$ :

$$
\operatorname{card} A_{0}=4, \quad \text { and } \quad \operatorname{card} A_{i+1}-\operatorname{card} A_{i}=4, \quad i=1,2, \ldots
$$

In Table 8 we present these subsets of the odd numbers $A_{i}$ from 1 to 1087 (for $n=0$ to $n=15$ ), which correspond to the squares of the odd numbers that are smaller or equal to $\sqrt{ } 1087$, that is 31 . In these subsets for easier distinction, the composites are encoded in 'turquoise' while the primes in 'black' colour.

By the term "wave number" mentioned in the sequence, we mean the number of waves which propagate through the particular subset. The first subset, 1 to $7(n=0)$, is not traversed by any wave. The second, 9 to $23,(n=1)$, is traversed by a wave that gives the multiples of 3 . The third, 25 to $47(n=2)$, by two waves that give the multiples of 3 and 5 , and so on.

According to the above, the conversion of some natural numbers to composites and the distribution of the arithmetic line is governed by specific rules that are uniform for all natural numbers. The procedure is deterministic and therefore independent of sample size.

From what we have presented here the validity of the statements below is obvious.

Sentence-1: The relation between the first term $x_{0}$ of every subset $A_{n}$ and its ascending number $n$ is: $x_{0}=(2 n+1)^{2}$. At the same time, the symbol $n$ represents also the number of waves that transverse the concrete subset $\left(A_{n}\right)$.

Sentence-2: Every new subset $A_{i}$ has 4 terms (odd numbers) more than its previous subset, $A_{i-1}$. See also $\operatorname{Eq}(27)$.

Sentence-3: In every new subset $A_{i}$ only one new wave acts, starting from $(2 n+1)^{2}$ and step (half wave) $2(2 n+1)$. Since the term $(2 n+1)$ is a prime number, every new wave creates composite numbers using the formula (21), that is, $(C N)_{(2 n+1)}=(2 n+1)^{2}+2(2 n+1) \mu$.

Sentence-4: Easily proved that for any $n$, in the concrete wave $\mu$ may take only the values $\mu=0,1$ and 2 (three intersections of the transverse wave with the axis $\mathrm{O} x$ for each new subset). Since the term $(2 n+1)$ is a composite 


\begin{tabular}{|c|}
\hline 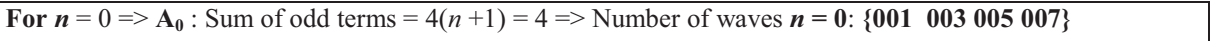 \\
\hline 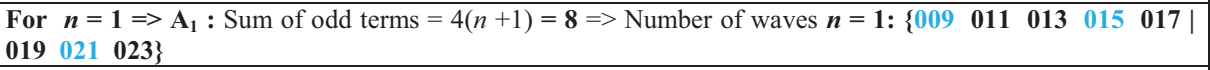 \\
\hline $\begin{array}{l}\text { For } \boldsymbol{n}=\mathbf{2} \Rightarrow \mathbf{A}_{2}: \text { Sum of odd terms }=4(n+1)=\mathbf{1 2}=>\text { Number of waves } \boldsymbol{n}=\mathbf{2} \\
\{025027 \mathbf{0 2 9} 031033|035 \mathbf{0 3 7} 039041043| 045047\}\end{array}$ \\
\hline $\begin{array}{l}\text { For } \boldsymbol{n}=\mathbf{3}=>\mathbf{A}_{\mathbf{3}}: \text { Sum of odd terms }=4(n+1)=\mathbf{1 6} \text { Number of waves } \boldsymbol{n}=\mathbf{3} \\
\{049051 \mathbf{0 5 3} 055057|\mathbf{0 5 9} \mathbf{0 6 1} 063065 \mathbf{0 6 7}| 069 \mathbf{0 7 1 0 7 3} 075077 \mid \mathbf{0 7 9}\end{array}$ \\
\hline $\begin{array}{l}\text { For } n=4 \Rightarrow \mathbf{A}_{\mathbf{4}}: \text { Sum of odd terms }=4(n+1)=\mathbf{2 0} \text { Number of waves } \boldsymbol{n}=\mathbf{4} \\
\{081 \mathbf{0 8 3} 085087 \mathbf{0 8 9}|091093095 \mathbf{0 9 7} 099| \mathbf{1 0 1} \mathbf{1 0 3} 105 \mathbf{1 0 7} \mathbf{1 0 9} \mid 111 \mathbf{1 1 3} 115117119\}\end{array}$ \\
\hline 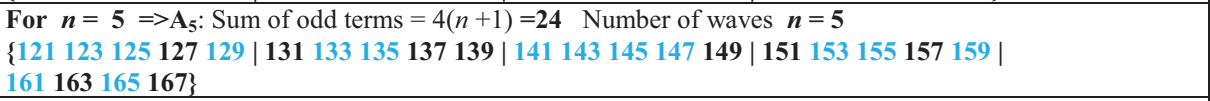 \\
\hline 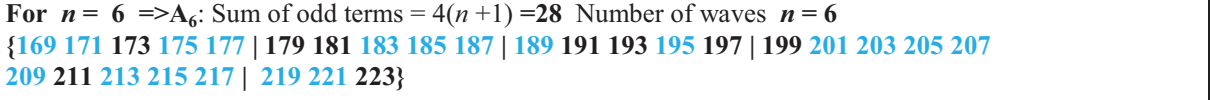 \\
\hline 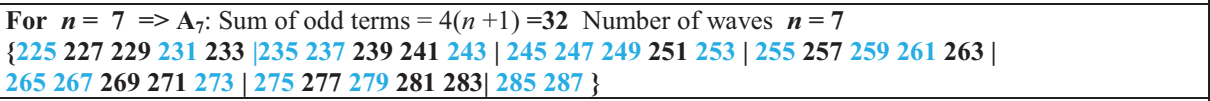 \\
\hline $\begin{array}{l}\text { For } \boldsymbol{n}=\mathbf{8} \Rightarrow \mathbf{A}_{\mathbf{8}}: \text { Sum of odd terms }=4(n+1)=\mathbf{3 6} \text { Number of waves } \boldsymbol{n}=\mathbf{8} \\
\{289291 \mathbf{2 9 3} 295297|299301303305 \mathbf{3 0 7 |} 309 \mathbf{3 1 1} \mathbf{3 1 3} 315 \mathbf{3 1 7}| 319321323325327 \\
329 \mathbf{3 3 1} 333335 \mathbf{3 3 7}|339341343345 \mathbf{3 4 7}| \mathbf{3 4 9} 351 \mathbf{3 5 3} 355357 \mid \mathbf{3 5 9}\}\end{array}$ \\
\hline 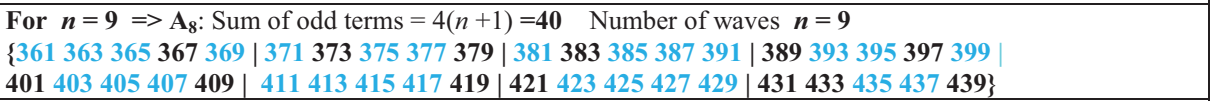 \\
\hline $\begin{array}{l}\text { For } \boldsymbol{n}=\mathbf{1 0}=>\mathbf{A}_{\mathbf{1 0}}: \text { Sum of odd terms }=4(n+1)=\mathbf{4 4} \text { Number of waves } \boldsymbol{n}=\mathbf{1 0} \\
\{441 \mathbf{4 4 3} 445447 \mathbf{4 4 9}|451453455 \mathbf{4 5 7} 459| \mathbf{4 6 1} \mathbf{4 6 3} 465 \mathbf{4 6 7} 469|471473475477 \mathbf{4 7 9}| \\
481483485 \mathbf{4 8 7} 489|\mathbf{4 9 1} 493495497 \mathbf{4 9 9}| 501 \mathbf{5 0 3} 505507 \mathbf{5 0 9}|511513515517519| \\
\mathbf{5 2 1} \mathbf{5 2 3} 525 \mathbf{5 2 7}\}\end{array}$ \\
\hline 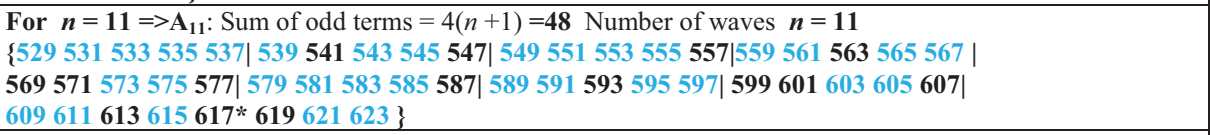 \\
\hline $\begin{array}{l}\text { For } \boldsymbol{n}=\mathbf{1 2}=>\mathbf{A}_{\mathbf{1 2}}: \text { Sum of odd terms }=4(n+1)=\mathbf{5 2} \text { Number of waves } \boldsymbol{n}=\mathbf{1 2} \\
\{625627629 \mathbf{6 3 1} 633|635637639 \mathbf{6 4 1} \mathbf{6 4 3}| 645 \mathbf{6 4 7} 649651 \mathbf{6 5 3} \mid 655657 \mathbf{6 5 9 6 6 1 6 6 3 |} \\
665667669671 \mathbf{6 7 3}|675 \mathbf{6 7 7} 679681 \mathbf{6 8 3}| 685687689 \mathbf{6 9 1} 693|695697699 \mathbf{7 0 1} 703| \\
705707 \mathbf{7 0 9} 711713 * 715717 \mathbf{7 1 9} 721723 * 725 \mathbf{7 2 7}\}\end{array}$ \\
\hline $\begin{array}{l}\text { For } \boldsymbol{n}=\mathbf{1 3} \Rightarrow \mathbf{A}_{\mathbf{1 3}}: \text { Sum of odd terms }=4(n+1)=\mathbf{5 6} \text { Number of waves } \boldsymbol{n}=\mathbf{1 3} \\
\{729731 \mathbf{7 3 3} 735737|\mathbf{7 3 9} 741 \mathbf{7 4 3} 745747| 749 \mathbf{7 5 1} 753755 \mathbf{7 5 7 | 7 5 9} \mathbf{7 6 1} 763765767 \mid \\
\mathbf{7 6 9} 771 \mathbf{7 7 3} 775777|779781783785 \mathbf{7 8 7 |} 789791793795 \mathbf{7 9 7 |} 799801803805807| \\
\mathbf{8 0 9 8 1 1} 813815817|819 \mathbf{8 2 1} \mathbf{8 2 3} 825 \mathbf{8 2 7} \mathbf{8 2 9} 831833835837| \mathbf{8 3 9}\}\end{array}$ \\
\hline 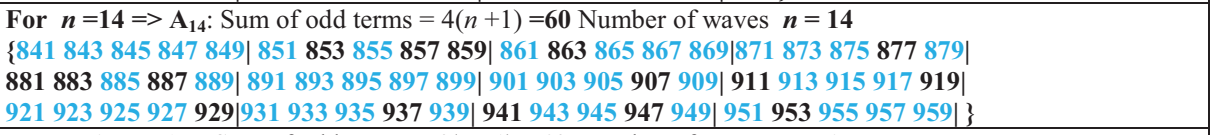 \\
\hline 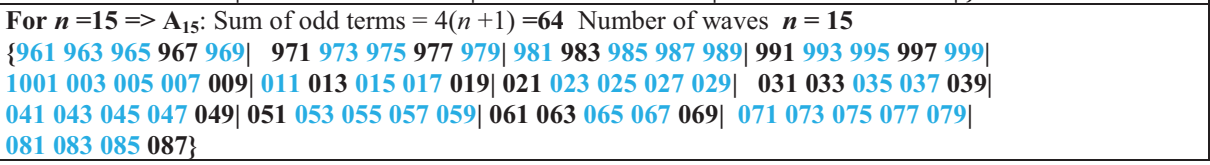 \\
\hline
\end{tabular}

Table 8: Subsets of odd numbers $A_{i}$ (from $n=0$ to $n=15$ ), the composites encoded in turquoise colour while the primes in black. The vertical bar $(\mid)$ appears only for visual purposes, every five numbers. 
number, it does not create new composites based on the formula: $(C N)_{(2 n+1)}=$ $(2 n+1)^{2}+2(2 n+1) \mu$.

Sentence-5: In this way the complex numbers are created, each new wave will form 0,1 , or at most 2 new composite numbers, in the first subset it acts. The value of $\mu=2$ coincides with the values that are multiples of 3 .

Sentence-6: From the above analysis, it is showed that the creation of composite numbers follows a uniform determinism, from the first up to the last subset $A_{i}$ we created, using the formula $A_{i}=\left\{x / x=(2 n+1)^{2}, x<(2 n+3)^{2}\right\}$ (24) . Therefore, both the crowd and the distribution of prime numbers will also be deterministically defined and uniform throughout the set of natural numbers.

Finding: Concerning the distribution of prime numbers, in the extended sample that we examined (and which we can expand indefinitely) is such that it follows very closely the probabilistic relationships (16) and this distribution is strictly deterministically defined for all of natural numbers, we have the right to extend the validity of (16) for the entire set of natural numbers.

\section{Extension of Equation (13a) for the Entire set of Natural Numbers}

Theorem 4. If we call $p(N)$ the function that counts the number of ordered pairs of primes that fulfill Goldbach's conjecture for a natural number $N$, this crowd is approximated by the formula:

$$
p(N)=N /[\log (N / 2)]^{2}
$$

Proof. Let us consider a sufficiently large even number $N$. According to the Prime Number Theorem (PNT), the primes that exist in the set from 1 to $N$, are approximated by:

$$
\pi(N)=N / \log (N)
$$

If we decompose this even number in ordered pairs of odd numbers according to equations (9) and (10), the odd numbers that correspond to the column $A$, and are in increasing order, will be all less than $N / 2$. Therefore, the primes $\alpha_{1}$, which exist in column $A$, will be given by:

$$
\alpha_{1}=\pi(N / 2)=(N / 2) /[\log (N / 2)] .
$$

As a result, the primes $\beta_{1}$ that exist in the column $B$, will be the subtraction of (30) from (29):

$$
\beta_{1}=N / \log (N)-(N / 2) / \log (N / 2) .
$$


But $N / \log (N)$ can be written as $N / \log [2 \cdot(N / 2)]$ or $N /[\log (N / 2)+\log 2]$, and for a sufficiently large $N$ the latter is approximated by $N / \log (N / 2)$, for which $\mathrm{Eq}(31)$ becomes:

$$
\beta_{1} \cong N / \log (N / 2)-(N / 2) / \log (N / 2)=(N / 2) /[\log (N / 2)] .
$$

Thus $\mathrm{Eq}(30)$ and $\mathrm{Eq}(32)$ suggest that, for very large even numbers $N$, the primes $\alpha_{1}$ in column $A$ and the primes $\beta_{1}$ in column $B$, tend to become equal $\left(\alpha_{1} \cong \beta_{1}\right)$.

From what we have mentioned for the crowd $n_{s}$ of ordered pairs in which the even number $N$ is decomposed, if it is put in the form $6 n-2$ and $6 n$ it will be $(N / 4)-1$, while if it is put in the form $6 n+2$ it will be $N / 4$. For very large $N$, we can consider all three cases with $n_{s}=N / 4$, without causing any serious mistake.

Substituting the above values of $\alpha_{1}, \beta_{1}$ and $n_{s}$ into $\mathrm{Eq}(13 \mathrm{a})$, we obtain:

$$
n_{p p} \cong(N / 2) /[\log (N / 2)]^{2} /(N / 4) \text {. }
$$

If both parts of $\mathrm{Eq}(33)$ are mupltiplied by $n_{s} \cong N / 4$, then $\mathrm{Eq}(28)$ is obtained. This completes the proof of Theorem 4.

The graphical representation of $\mathrm{Eq}(28)$ is illustrated by the green line in Figure 7.

In Figure 7 we observe that, the graph of $\mathrm{Eq}(28)$ representing the number of verifications of Goldbach's conjecture is below the corresponding number using $\mathrm{Eq}(13 \mathrm{a})$ and is located just below the average line of true verifications (red line). Note that the red line of Figure 7 does not correspond to the red line in Figure 2, but the union of sets of values corresponding together to the purple and red line in Fig.2, which corresponds to the sum of true pairs. This is a natural consequence of the fact that the number of prime numbers calculated from (PNT), is below the true number of primes $\pi(N)$, as shown in Figure 8.

\section{Monotonically Increasing Function}

Theorem 5. The estimator of the verifications $p(N)$ of Goldbach's conjecture is a monotonically increasing function of $N$.

Proof. We consider the continuous function $p(x)=x /[\log (x / 2)]^{2}$. The first and second derivatives are given by:

$$
p^{\prime}(x)=[\log (x / 2)-2] /[\log (x / 2)]^{3}
$$




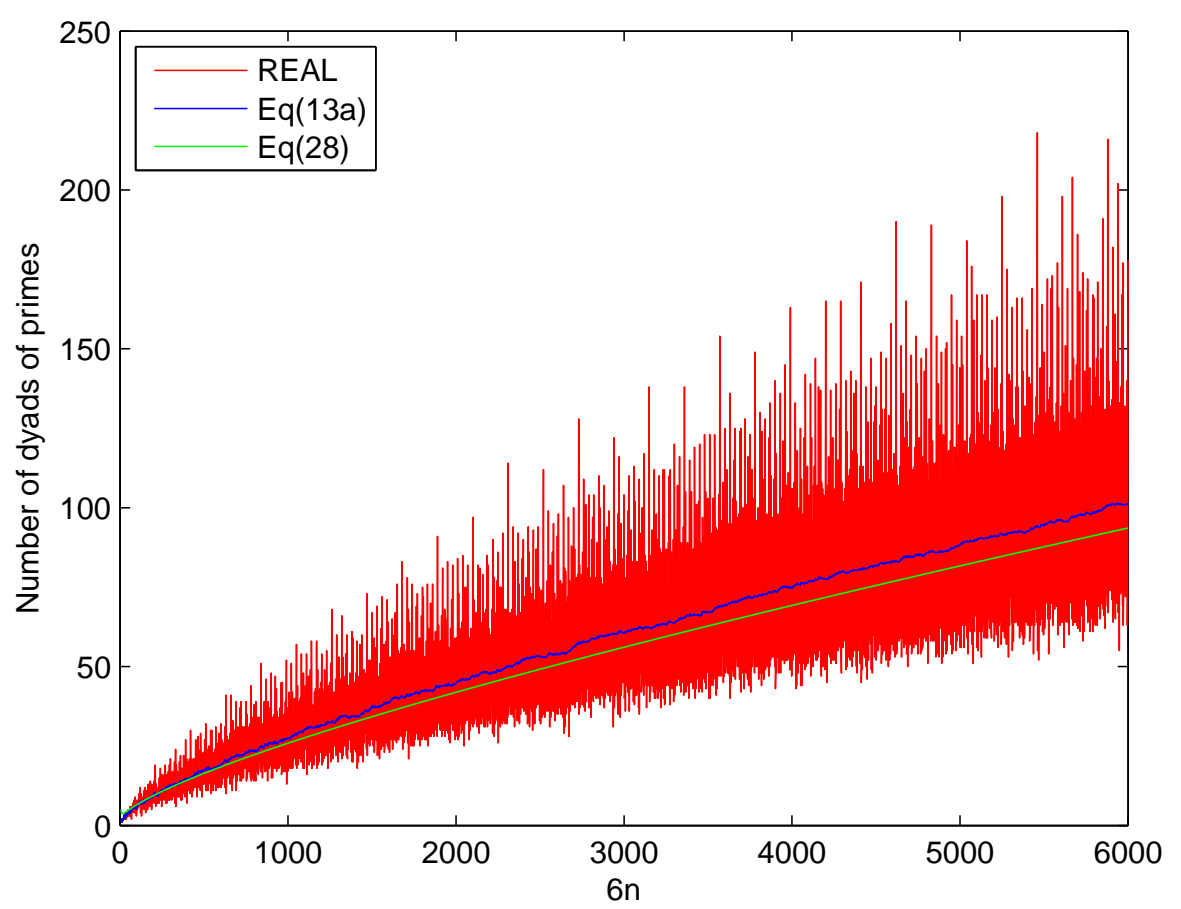

Figure 7: Comparison between the true number of pairs of prime numbers that fulfill Goldbach's conjecture and the approximations using eq(13a) and eq(28).

$$
p^{\prime \prime}(x)=-2[\log (x / 2)-3] / x[\log (x / 2)]^{4}
$$

Since for the first derivative it obviously holds that:

$$
p^{\prime}(x)>0, \quad \forall x>2 e^{2}
$$

while the second derivative never becomes zero :

$$
p^{\prime \prime}(x)>0, \quad \forall x>2 e^{3},
$$

it is finally concluded that the function $p(x)$ is monotonically increasing and convex. 


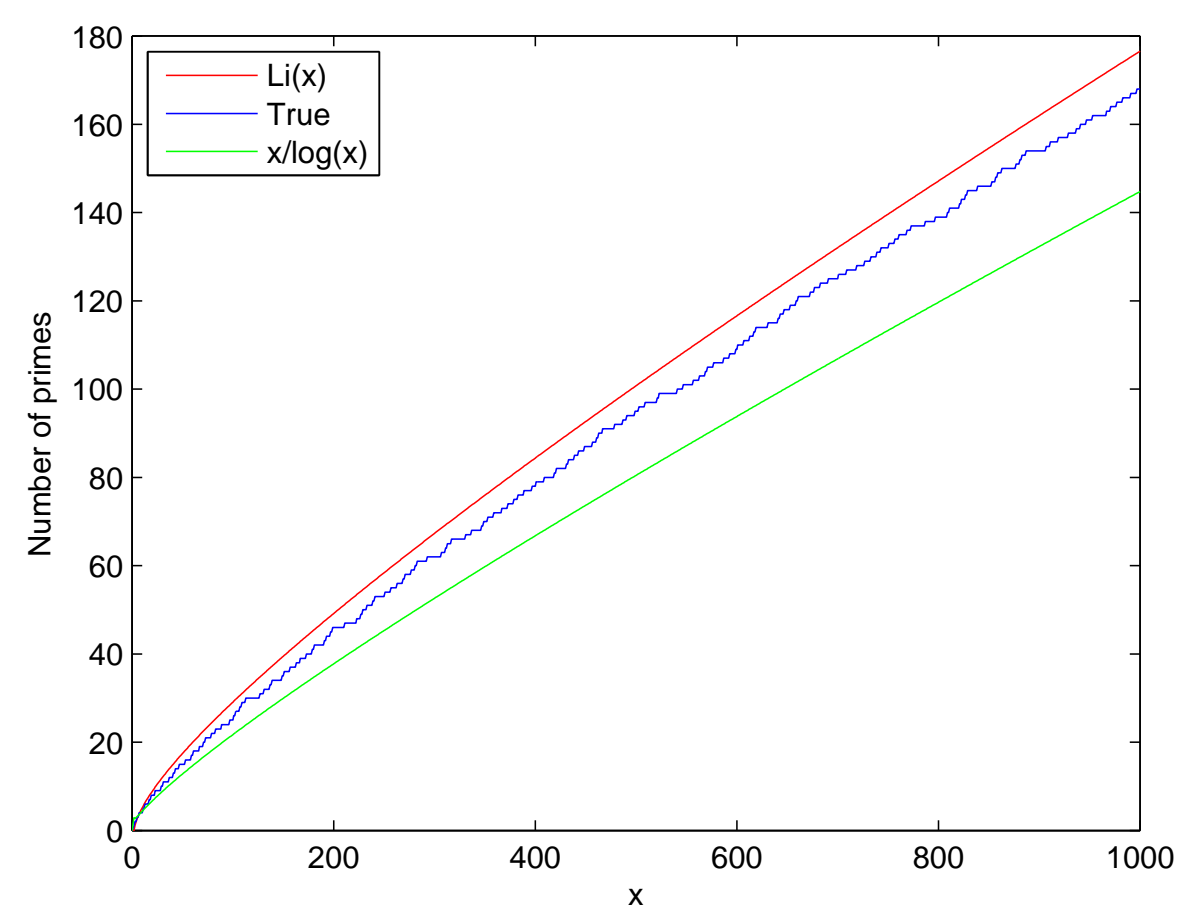

Figure 8: Prime Number Theorem [red line: according to the function $\operatorname{Li}(x)$, Blue line: true number, Green line: according to $x / \log (x)]$.

\section{Discussion}

This paper includes ten original points as follows:

1) The definition of six equivalence classes in $\mathbb{N}$ through $\operatorname{Eq}(3)$ is original. The profit from their definition is that, in this way we achieved to condense the feasible set of primes and their multiples in one-third, through the expression $6 \lambda \pm 1$.

2) Concerning the even numbers themselves, we divided them into three subsets, i.e. like a basic cell $(6 n-2,6 n, 6 n+2)$. These subsets were further put as a sum of two odd numbers of the form $6 \lambda \pm 1$ (equations 9,10 ).

3) For the three even numbers that appear in the above cell $(6 n-2,6 n, 6 n+$ 2 ), we found that for odd numbers in the form of the centered numbers, i.e. $6 n$, the probability to fulfill Goldbach's conjecture is about twice as that for either $6 n-2$ or $6 n+2$. 
4) The first three theorems (Theorem-1, Theorem-2 and Theorem-3), through which we determine the number of couples of odd numbers (not necessarily primes) in which the even numbers are decomposed, are new.

5) In contrast to the established opinion that the distribution of the primes along the arithmetic line is random, we depicted that it is a severely deterministic 'phenomenon'.

6) We found that the density and distribution of prime numbers ensure that $\alpha_{1}$ and $\beta_{1}$ increase in such a way that, the product $\left(\alpha_{1} \beta_{1}\right)$ increases faster than what the total number of couples $n_{s}$ increases, thus resulting in a continuously increasing number of verifications of Goldbach's conjecture.

7) We refined a rule (sieve) to create Composite Numbers, which is based on waves of prescribed starting point and predetermined wave-length (equation(21)). We established that between all the aforementioned waves there are some numbers that remain untouched. This means that there are natural numbers $N_{P}$ that remain primes, obviously because the following relationship is fulfilled: $\left(N_{P}-P_{i}^{2}\right) / 2 P_{i} \mu_{i}$, where $P_{i}$ are successive primes $\leq \sqrt{ } N$ such as 5,7 , 11, etc. Also, using $\operatorname{Eq}(22)$ is helpful to make the decision whether any arbitrary natural number $N$ is prime or not.

8) The extension of $\mathrm{Eq}(13 \mathrm{a})$ to the entire set of natural numbers is novel.

9) The closed form expression for the estimation of pairs that fulfill Goldbach's conjecture.

10) We proved that the function $p(N)$ is monotonically increasing.

\section{Conclusion}

We proved a stronger sentence than the original Goldbach's conjecture, which can be formulated as follows:

Final Sentence. The strictly predetermined distribution of primes along the arithmetic line is such as to ensure that as we move towards larger and larger even numbers $N$, the growth of the product $\left(\alpha_{1} \beta_{1}\right)$ is greater than the growth rate of the number of ordered pairs of odd numbers $n_{s}$ in which the even number under question is analyzed using equations (9) and (10), where $\alpha_{1}$ is the number of prime numbers in column $A$ and $\beta_{1}$ is the number of prime numbers in column $B$ of ordered pairs. The direct result of this property of prime numbers is that:

Not only Goldbach's conjecture is true, but the number of verifications has a clearly increasing trend, as we move to larger even numbers, which is determined by relation (28). 


\section{References}

[1] Christian Goldbach, Letter to L. Euler, June 7 (1742).

[2] David Hilbert, Mathematical Problems, Bulletin of the American Mathematical Society, 8, No. 10 (1902), 437-479. Earlier publications (in the original German) appeared in Göttinger Nachrichten (1900), 253-297, and Archiv der Mathematik und Physik, 3dser., 1 (1901), 44-63 and 213-237.

[3] János Pintz, Landau's problems on primes, Journal de théorie des nombres de Bordeaux, 21, No. 2 (2009) 357-404.

[4] Richard K. Guy, Unsolved problems in number theory, Third Edition, Springer-Verlag, Berlin (2004).

[5] G. H. Hardy and J. E. Littlewood, Some problems of 'Partitio numerorum'; III: On the expression of a number as a sum of primes, Acta Mathematica, 44, No. 1 (1923), 1-70.

[6] Viggo Brun, Le crible d'Eratosthene et le theoreme de Goldbach, C. R. Acad. Sci., Paris, 168 (1919), 544-546.

[7] L. G. Schnirelman, On additive properties of numbers. Izv. Donsk. Politehn. Inst. 14 (1930), 328 (In Russian); Also: L. G. Schnirelman, Über additive Eigenschaften von Zahlen, Math. Ann., 107 (1933), 649-690.

[8] I. M. Vinogradov, Some theorems in analytic theory of numbers, Dokl. Akad. Nauk SSSR, 4 (1934), 185-187. Also: I. M. Vinogradov, Elements of Number Theory, Dover Publications, Mineola, NY (2003).

[9] K. G. Borozdkin, On a problem of Vinogradov's constant, Trudy Mat. Soc, SSSR, 1 (1956), 3.

[10] Jingrun Chen, On the representation of a large even integer as the sum of a prime and the product of at most 2 primes, it Kexue Tongbao, 17 (1966), 385-386.

[11] M. C. Liu, T. Z. Wang, On the Vinogradov's bound in the three primes Goldbach conjecture, Acta Arithmetica, 105, No. 2 (2002), 133-175.

[12] N. Lygeros, F. Morain, O. Rozier, http://www.lix.polytechnique.fr/ morain /Primes/myprimes.html

[13] Tomás Oliveira e Silva, http://www.ieeta.pt/ tos/goldbach.html 
[14] J.-M. Deshouillers, G. Effinger, H. Te Riele and D. Zinoviev, A complete Vinogradov 3-primes theorem under the Riemann hypothesis. Electronic Research Announcements of the American Mathematical Society, 3, No. 15 (1997), 99-104.

[15] Leszek Kaniecki, On Šnirelman's constant under the Riemann hypothesis, Acta Arithmetica, 4 (1995), 361-374.

[16] Yuan Wang, Goldbach Conjecture, 2nd edn., World Scientific Publishing, New Jersey, Singapore (2000).

[17] Nikolai G. Chudakov, On the Goldbach problem, Doklady Akademii Nauk SSSR, 17 (1937), 335-338.

[18] J. G. Van der Corput, Sur l'hypothèse de Goldbach, Proc. Akad. Wet. Amsterdam, 41 (1938), 76-80.

[19] T. Estermann, On Goldbach's problem: proof that almost all even positive integers are sums of two primes, Proc. London Math. Soc., Sér. 2, 44 (1938), 307-314.

[20] Ramar, Olivier, On Šnirel'man's constant, Annali della Scuola Normale Superiore di Pisa - Classe di Scienze, Sér. 4, 22, No. 4 (1995), 645-706.

[21] Matti K. Sinisalo, Checking the Goldbach Conjecture up to $4 \cdot 10^{11}$, Mathematics of Computation, 61, No. 204 (1993), 931-934.

[22] J. R. Chen, On the representation of a larger even integer as the sum of a prime and the product of at most two primes, Sci. Sinica, 16 (1973), $157-176$.

[23] H.L. Montgomery, R.C. Vaughan, The exceptional set in Goldbach's problem, Acta Arith., 27 (1975), 353-370.

[24] Yu. V. Linnik, Prime numbers and powers of two, Collection of articles. To the sixtieth birthday of academician Ivan Matveevich Vinogradov, Trudy Mat. Inst. Steklov., 38, Acad. Sci. USSR, Moscow (1951), 152-169.

[25] D. R. Heath-Brown, J. C. Puchta, Integers represented as a sum of primes and powers of two, Asian Journal of Mathematics, 6, No. 3 (2002), 535-565.

[26] J.Pintz, I. Z. Ruzsa, On Linnik's approximation to Goldbach's problem, I, Acta Arithmetica, 109, No. 2 (2003), 169-194. 
[27] Jörg Richstein, Verifying the Goldbach conjecture up to $4 \cdot 10^{14}$, Mathematics of Computation, 70, No. 236 (2000), 1745-1749.

[28] I. Mittas, Generalization of Goldbach's conjecture and some special cases, Italian Journal of Pure and Applied Mathematics, 27 (2010), 241-254.

[29] http://en.wikipedia.org/wiki/Goldbach's_weak_conjecture

[30] Eric W. Weisstein, Goldbach Conjecture. From MathWorld - A Wolfram Web Resource, http://mathworld.wolfram.com/GoldbachConjecture.html

[31] http://jtnb.cedram.org/item?id=JTNB_2009__21_2_357_0

[32] http://jtnb.cedram.org/cedram-bin/article/JTNB_2009__21_2_357_0.pdf

[33] Neil Sheldon, A statistician's approach to Goldbachs Conjecture, Teaching Statistics 25, No. 1 (2003), 12-13.

[34] Song Y. Yan, A simple verification method for the Goldbach conjecture, International Journal of Mathematical Education in Science and Technology, 25, No. 5 (1994), 681-688.

[35] John Derbyshire, Prime Obsession: Bernhard Riemann and the Greatest Unsolved Problem in Mathematics, Joseph Henry Press, Washington, D.C. (2003).

[36] Apostolos Doxiadis, Uncle Petros and Goldbach's Conjecture, Bloomsbury, New York (2001).

[37] Marcus du Sautoy, The Music of the Primes: Searching to Solve the Greatest Mystery in Mathematics, HarperCollins Publishers, New York (2003).

[38] John Harrison, Formalizing an Analytic Proof of the Prime Number Theorem, J Autom Reasoning, 43 (2009), 243-261.

[39] D. J. Newman, Simple Analytic Proof of the Prime Number Theorem, The American Mathematical Monthly, 87, No. 9 (1980), 693-696. 


\section{Appendix A}

Prime Number Theorem (PNT). Let $\pi(x)$ be the prime-counting function that gives the number of primes less than or equal to $x$, for any real number $x$. The prime number theorem then states that the limit of the quotient of the two functions $\pi(x)$ and $x / \ln (x) \equiv x / \log (x)$ as $x$ approaches infinity is 1 , which is expressed by the formula:

$$
\lim _{x \rightarrow \infty} \frac{\pi(x)}{x / \log x}=1
$$

known as "the asymptotic law of distribution of prime numbers". Using asymptotic notation this result can be restated as:

$$
\pi(x) \simeq x / \log (x)
$$

This theorem does not say anything about the limit of the difference of the two functions as $x$ approaches infinity. Instead, the theorem states that $x / \ln (x)$ approximates $\pi(x)$ in the sense that the relative error of this approximation approaches 0 as $x$ approaches infinity.

The prime number theorem is equivalent to the statement that the $n$th prime number $p_{n}$ is approximately equal to $n \ln (n)$, again with the relative error of this approximation approaching 0 as $n$ approaches infinity.

For analytical proofs we refer to $[38,39]$ among others.

Corrolary. The average density of primes is given by: $\pi(N) \sim 1 / \log N$. Obviously, it decreases by increasing $N$. As a result, the density of primes in the column $A$ is greater than that of column $B$ and at the same time the density of primes in the two columns is different. 
